\title{
ON THE STRONGLY AMBIGUOUS CLASSES OF SOME BIQUADRATIC NUMBER FIELDS
}

\author{
ABDELMALEK AZIZI, ABDELKADER ZEKHNINI, AND MOHAMMED TAOUS
}

\begin{abstract}
We study the capitulation of ideal classes in an infinite family of imaginary bicyclic biquadratic number fields consisting of fields $\mathrm{k}=\mathbb{Q}(\sqrt{2 p q}, i)$, where $i=\sqrt{-1}$ and $p \equiv-q \equiv 1(\bmod 4)$ are different primes. For each of the three quadratic extensions $\mathbb{K} / \mathbb{k}$ inside the absolute genus field $\mathbb{k}^{(*)}$ of $\mathbb{k}$, we compute the capitulation kernel of $\mathbb{K} / \mathbb{k}$. Then we deduce that each strongly ambiguous class of $\mathrm{k} / \mathbb{Q}(i)$ capitulates already in $\mathbb{k}^{(*)}$, which is smaller than the relative genus field $(\mathrm{k} / \mathbb{Q}(i))^{*}$.
\end{abstract}

\section{INTRODUCTION}

Let $k$ be an algebraic number field and let $\mathbf{C l}_{2}(k)$ denote its 2-class group, that is the 2-Sylow subgroup of the ideal class group, $\mathbf{C l}(k)$, of $k$. We denote by $k^{(*)}$ the absolute genus field of $k$. Suppose $F$ is a finite extension of $k$, then we say that an ideal class of $k$ capitulates in $F$ if it is in the kernel of the homomorphism

$$
J_{F}: \mathbf{C l}(k) \longrightarrow \mathbf{C l}(F)
$$

induced by extension of ideals from $k$ to $F$. An important problem in Number Theory is to determine explicitly the kernel of $J_{F}$, which is usually called the capitulation kernel. If $F$ is the relative genus field of a cyclic extension $K / k$, which we denote by $(K / k)^{*}$ and that is the maximal unramified extension of $K$ which is obtained by composing $K$ and an abelian extension over $k$, F. Terada states in [11] that all the ambiguous ideal classes of $K / k$, which are classes of $K$ fixed under any element of $\operatorname{Gal}(K / k)$, capitulate in $(K / k)^{*}$. If $F$ is the absolute genus field of an abelian extension $K / \mathbb{Q}$, then H. Furuya confirms in [13] that every strongly ambiguous class of $K / \mathbb{Q}$, that is an ambiguous ideal class containing at least one ideal invariant under any element of $\operatorname{Gal}(K / \mathbb{Q})$, capitulates in $F$. In this paper, we construct a family of number fields $k$ for which $\mathbf{C} l_{2}(k) \simeq(2,2,2)$ and all the strongly ambiguous classes of $k / \mathbb{Q}(i)$ capitulate in $k^{(*)} \varsubsetneqq(k / \mathbb{Q}(i))^{*}$.

Let $p$ and $q$ be different primes, $\mathbb{k}=\mathbb{Q}(\sqrt{2 p q}, i)$ and $\mathbb{K}$ be an unramified quadratic extension of $\mathbb{k}$ that is abelian over $\mathbb{Q}$. Denote by $A m_{s}(\mathbb{k} / \mathbb{Q}(i))$ the group of the strongly ambiguous classes of $\mathbb{k} / \mathbb{Q}(i)$. In [1], the first author has studied the capitulation problem in $\mathbb{K} / \mathbb{k}$ assuming $p \equiv-q \equiv 1(\bmod 4)$ and

2010 Mathematics Subject Classification. 11R11, 11R16, 11R20, 11R27, 11R29, 11R37.

Key words and phrases. absolute and relative genus fields, fundamental systems of units, 2-class group, capitulation, quadratic fields, biquadratic fields, multiquadratic CM-fields. 
$\mathbf{C} l_{2}(\mathbb{k}) \simeq(2,2)$. On the other hand, in [6], we have dealt with the same problem assuming $p \equiv q \equiv 1(\bmod 4)$, and in [7], we have studied the capitulation problem of the 2-ideal classes of $\mathrm{k}$ in its fourteen unramified extensions, within the first Hilbert 2-class field of $\mathbb{k}$, assuming $p \equiv q \equiv 5(\bmod 8)$. It is the purpose of the present article to pursue this research project further for all types of $\mathbf{C} l_{2}(\mathbb{k})$, assuming $p \equiv-q \equiv 1(\bmod 4)$, we compute the capitulation kernel of $\mathbb{K} / \mathbb{k}$ and we deduce that $\mathrm{A}_{s}(\mathbb{k} / \mathbb{Q}(i)) \subseteq \operatorname{ker} J_{\mathbb{k}(*)}$. As an application we will determine these kernels when $\mathbf{C} l_{2}(\mathrm{k})$ is of type $(2,2,2)$.

Let $k$ be a number field, during this paper, we adopt the following notations:

- $p \equiv-q \equiv 1(\bmod 4)$ are different primes.

- $\mathbb{k}$ : denotes the field $\mathbb{Q}(\sqrt{2 p q}, \sqrt{-1})$.

- $\kappa_{K}$ : the capitulation kernel of an unramified extension $K / \mathbb{k}$.

- $\mathcal{O}_{k}$ : the ring of integers of $k$.

- $E_{k}$ : the unit group of $\mathcal{O}_{k}$.

- $W_{k}$ : the group of roots of unity contained in $k$.

- F.S.U : the fundamental system of units.

- $k^{+}$: the maximal real subfield of $k$, if it is a CM-field.

- $Q_{k}=\left[E_{k}: W_{k} E_{k^{+}}\right]$is Hasse's unit index, if $k$ is a CM-field.

- $q(k / \mathbb{Q})=\left[E_{k}: \prod_{i=1}^{s} E_{k_{i}}\right]$ is the unit index of $k$, if $k$ is multiquadratic, where $k_{1}, \ldots, k_{s}$ are the quadratic subfields of $k$.

- $k^{(*)}$ : the absolute genus field of $k$.

- $\mathrm{Cl}_{2}(k)$ : the 2-class group of $k$.

- $i=\sqrt{-1}$.

- $\epsilon_{m}$ : the fundamental unit of $\mathbb{Q}(\sqrt{m})$, if $m>1$ is a square-free integer.

- $N(a)$ : denotes the absolute norm of a number a i.e. $N_{k / \mathbb{Q}}(a)$, where $k=\mathbb{Q}(\sqrt{a})$.

- $x \pm y$ means $x+y$ or $x-y$ for some numbers $x$ and $y$.

\section{Preliminary results}

Let us first collect some results that will be useful in what follows.

Let $k_{j}, 1 \leq j \leq 3$, be the three real quadratic subfields of a biquadratic bicyclic real number field $K_{0}$ and $\epsilon_{j}>1$ be the fundamental unit of $k_{j}$. Since $\alpha^{2} N_{K_{0} / \mathbf{Q}}(\alpha)=\prod_{j=1}^{3} N_{K_{0} / k_{j}}(\alpha)$ for any $\alpha \in K_{0}$, the square of any unit of $K_{0}$ is in the group generated by the $\epsilon_{j}{ }^{\prime}$ s, $1 \leq j \leq 3$. Hence, to determine a system of fundamental units of $K_{0}$ it suffices to determine which of the units in $B:=\left\{\epsilon_{1}, \epsilon_{2}, \epsilon_{3}, \epsilon_{1} \epsilon_{2}, \epsilon_{1} \epsilon_{3}, \epsilon_{2} \epsilon_{3}, \epsilon_{1} \epsilon_{2} \epsilon_{3}\right\}$ are squares in $K_{0}$ (see [15] or [19]). Put $K=K_{0}(i)$, then to determine a F.S.U of $K$, we will use the following result (see [2, p.18]) that the first author has deduced from a theorem of Hasse [14, §21, Satz $15]$. 
Lemma 2.1. Let $n \geq 2$ be an integer and $\xi_{n} a 2^{n}$-th primitive root of unity, then

$$
\xi_{n}=\frac{1}{2}\left(\mu_{n}+\lambda_{n} i\right), \quad \text { where } \begin{aligned}
\mu_{n} & =\sqrt{2+\mu_{n-1}}, \quad \lambda_{n}=\sqrt{2-\mu_{n-1}}, \\
\mu_{2} & =0, \lambda_{2}=2 \quad \text { and } \quad \mu_{3}=\lambda_{3}=\sqrt{2} .
\end{aligned}
$$

Let $n_{0}$ be the greatest integer such that $\xi_{n_{0}}$ is contained in $K,\left\{\epsilon_{1}^{\prime}, \epsilon_{2}^{\prime}, \epsilon_{3}^{\prime}\right\}$ a F.S.U of $K_{0}$ and $\epsilon$ a unit of $K_{0}$ such that $\left(2+\mu_{n_{0}}\right) \epsilon$ is a square in $K_{0}$ (if it exists). Then a F.S.U of $K$ is one of the following systems:

(1) $\left\{\epsilon_{1}^{\prime}, \epsilon_{2}^{\prime}, \epsilon_{3}^{\prime}\right\}$ if $\epsilon$ does not exist,

(2) $\left\{\epsilon_{1}^{\prime}, \epsilon_{2}^{\prime}, \sqrt{\xi_{n_{0}} \epsilon}\right\}$ if $\epsilon$ exists; in this case $\epsilon=\epsilon_{1}^{\prime i_{1}} \epsilon_{2}^{\prime i_{2}} \epsilon_{3}^{\prime}$, where $i_{1}, i_{2} \in\{0,1\}$ (up to a permutation).

Lemma 2.2 ([1], Lemma 5). Let $d>1$ be a square-free integer and $\epsilon_{d}=x+y \sqrt{d}$, where $x, y$ are integers or semi-integers. If $N\left(\epsilon_{d}\right)=1$, then $2(x+1), 2(x-1)$, $2 d(x+1)$ and $2 d(x-1)$ are not squares in $\mathbb{Q}$.

Lemma 2.3 ([1], Lemma 6$)$. Let $q \equiv-1(\bmod 4)$ be a prime and $\epsilon_{q}=x+y \sqrt{q}$ be the fundamental unit of $\mathbb{Q}(\sqrt{q})$. Then $x$ is an even integer, $x \pm 1$ is a square in $\mathbb{N}$ and $2 \epsilon_{q}$ is a square in $\mathbb{Q}(\sqrt{q})$.

Lemma 2.4 ([1], Lemma 7). Let $p$ be an odd prime and $\epsilon_{2 p}=x+y \sqrt{2 p}$. If $N\left(\epsilon_{2 p}\right)=1$, then $x \pm 1$ is a square in $\mathbb{N}$ and $2 \epsilon_{2 p}$ is a square in $\mathbb{Q}(\sqrt{2 p})$.

Lemma 2.5 ([2, 3.(1) p.19). Let $d>2$ be a square-free integer and $k=\mathbb{Q}(\sqrt{d}, i)$, put $\epsilon_{d}=x+y \sqrt{d}$.

(1) If $N\left(\epsilon_{d}\right)=-1$, then $\left\{\epsilon_{d}\right\}$ is a F.S.U of $k$.

(2) If $N\left(\epsilon_{d}\right)=1$, then $\left\{\sqrt{i \epsilon_{d}}\right\}$ is a F.S.U of $k$ if and only if $x \pm 1$ is a square in $\mathbb{N}$ i.e. $2 \epsilon_{d}$ is a square in $\mathbb{Q}(\sqrt{d})$. Else $\left\{\epsilon_{d}\right\}$ is a F.S.U of $k$ (this result is also in [20]).

\section{F.S.U OF SOME CM-FIELDS}

As $\mathbb{k}=\mathbb{Q}(\sqrt{2 p q}, i)$, so $\mathbb{k}$ admits three unramified quadratic extensions that are abelian over $\mathbb{Q}$, which are $\mathbb{K}_{1}=\mathbb{k}(\sqrt{p})=\mathbb{Q}(\sqrt{p}, \sqrt{2 q}, i), \mathbb{K}_{2}=\mathbb{k}(\sqrt{q})=$ $\mathbb{Q}(\sqrt{q}, \sqrt{2 p}, i)$ and $\mathbb{K}_{3}=\mathbb{k}(\sqrt{2})=\mathbb{Q}(\sqrt{2}, \sqrt{p q}, i)$. Put $\epsilon_{2 p q}=x+y \sqrt{2 p q}$, the first author has given in [1] the F.S.U's of these three fields, if $2 \epsilon_{2 p q}$ is not a square in $\mathbb{Q}(\sqrt{2 p q})$ i.e. $x+1$ and $x-1$ are not squares in $\mathbb{N}$. In what follows, we determine the F.S.U's of $\mathbb{K}_{j}, 1 \leq j \leq 3$, in all cases.

3.1. F.S.U of the field $\mathbb{K}_{1}$. Let $\mathbb{K}_{1}=\mathbb{k}(\sqrt{p})=\mathbb{Q}(\sqrt{p}, \sqrt{2 q}, i)$.

Proposition 3.1. Keep the previous notations, then $Q_{\mathbb{K}_{1}}=2$ and just one of the following two cases holds:

(1) If $x \pm 1$ or $p(x \pm 1)$ is a square in $\mathbb{N}$, then $\left\{\epsilon_{p}, \epsilon_{2 q}, \sqrt{\epsilon_{2 q} \epsilon_{2 p q}}\right\}$ is a F.S.U of $\mathbb{K}_{1}^{+}$and that of $\mathbb{K}_{1}$ is $\left\{\epsilon_{p}, \sqrt{i \epsilon_{2 q}}, \sqrt{\epsilon_{2 q} \epsilon_{2 p q}}\right\}$.

(2) If $2 p(x \pm 1)$ is a square in $\mathbb{N}$, then $\left\{\epsilon_{p}, \epsilon_{2 q}, \sqrt{\epsilon_{2 p q}}\right\}$ is a F.S.U of $\mathbb{K}_{1}^{+}$and that of $\mathbb{K}_{1}$ is $\left\{\epsilon_{p}, \sqrt{i \epsilon_{2 q}}, \sqrt{\epsilon_{2 p q}}\right\}$. 
Proof. As $p \equiv 1(\bmod 4)$, then $\epsilon_{p}$ is not a square in $\mathbb{K}_{1}^{+}$; but $\epsilon_{2 p q}$ and $\epsilon_{2 q} \epsilon_{2 p q}$ can be. Moreover, according to Lemma 2.4, $2 \epsilon_{2 q}$ is a square in $\mathbb{Q}(\sqrt{2 q})$. On the other hand, we know that $N\left(\epsilon_{2 p q}\right)=1$, then $(x \pm 1)(x \mp 1)=2 p q y^{2}$. Hence, by Lemma 2.2 and according to the decomposition uniqueness in $\mathbb{Z}$, there are three possibilities: $x \pm 1$ or $p(x \pm 1)$ or $2 p(x \pm 1)$ is a square in $\mathbb{N}$, the only remaining case is the first one. If $x \pm 1$ is a square in $\mathbb{N}$ (for the other cases see [1]), then, by Lemma 2.5, $2 \epsilon_{2 p q}$ is a square in $\mathbb{K}_{1}$. Consequently, $\sqrt{\epsilon_{2 q} \epsilon_{2 p q}} \in \mathbb{K}_{1}^{+}$; hence $\left\{\epsilon_{p}, \epsilon_{2 q}, \sqrt{\epsilon_{2 q} \epsilon_{2 p q}}\right\}$ is a F.S.U of $\mathbb{K}_{1}^{+}$, and since $2 \epsilon_{2 q}$ is a square in $\mathbb{K}_{1}^{+}$, so Lemma 2.1 yields that $\left\{\epsilon_{p}, \sqrt{i \epsilon_{2 q}}, \sqrt{\epsilon_{2 q} \epsilon_{2 p q}}\right\}$ is a F.S.U of $\mathbb{K}_{1}$. Thus $Q_{\mathbb{K}_{1}}=2$.

3.2. F.S.U of the field $\mathbb{K}_{2}$. Let $\mathbb{K}_{2}=\mathbb{k}(\sqrt{q})=\mathbb{Q}(\sqrt{q}, \sqrt{2 p}, i)$.

Proposition 3.2. Keep the previous notations, then $Q_{\mathbb{K}_{2}}=2$.

(1) Assume that $N\left(\epsilon_{2 p}\right)=1$. Then just one of the following two cases holds.

(i) If $x \pm 1$ or $2 p(x \pm 1)$ is a square in $\mathbb{N}$, then $\left\{\sqrt{\epsilon_{q} \epsilon_{2 p}}, \sqrt{\epsilon_{q} \epsilon_{2 p q}}, \sqrt{\epsilon_{2 p} \epsilon_{2 p q}}\right\}$ is a F.S.U of $\mathbb{K}_{2}^{+}$and that of $\mathbb{K}_{2}$ is $\left\{\sqrt{i \epsilon_{q}}, \sqrt{i \epsilon_{2 p}}, \sqrt{i \epsilon_{2 p q}}\right\}$.

(ii) If $p(x \pm 1)$ is a square in $\mathbb{N}$, then $\left\{\epsilon_{q}, \sqrt{\epsilon_{q} \epsilon_{2 p}}, \sqrt{\epsilon_{2 p q}}\right\}$ is a F.S.U of $\mathbb{K}_{2}^{+}$ and that of $\mathbb{K}_{2}$ is $\left\{\sqrt{i \epsilon_{q}}, \sqrt{i \epsilon_{2 p}}, \sqrt{\epsilon_{2 p q}}\right\}$.

(2) Assume that $N\left(\epsilon_{2 p}\right)=-1$. Then just one of the following two cases holds.

(i) If $x \pm 1$ or $2 p(x \pm 1)$ is a square in $\mathbb{N}$, then $\left\{\epsilon_{q}, \epsilon_{2 p}, \sqrt{\epsilon_{q} \epsilon_{2 p q}}\right\}$ is a F.S.U of $\mathbb{K}_{2}^{+}$and that of $\mathbb{K}_{2}$ is $\left\{\sqrt{i \epsilon_{q}}, \epsilon_{2 p}, \sqrt{\epsilon_{q} \epsilon_{2 p q}}\right\}$.

(ii) If $p(x \pm 1)$ is a square in $\mathbb{N}$, then $\left\{\epsilon_{q}, \epsilon_{2 p}, \sqrt{\epsilon_{2 p q}}\right\}$ is a F.S.U of $\mathbb{K}_{2}^{+}$ and that of $\mathbb{K}_{2}$ is $\left\{\sqrt{i \epsilon_{q}}, \epsilon_{2 p}, \sqrt{\epsilon_{2 p q}}\right\}$.

Proof. According to Lemma 2.5, if $x \pm 1$ is a square in $\mathbb{N}$, then $2 \epsilon_{2 p q}$ is a square in $\mathbb{Q}(\sqrt{2 p q})$. Moreover, Lemma 2.3 implies that $2 \epsilon_{q}$ is also a square in $\mathbb{Q}(\sqrt{q})$.

(1) If $N\left(\epsilon_{2 p}\right)=1$, then Lemma 2.4 yields that $2 \epsilon_{2 p}$ is a square in $\mathbb{Q}(\sqrt{2 p})$, thus $\epsilon_{2 p} \epsilon_{2 p q}, \epsilon_{q} \epsilon_{2 p q}$ and $\epsilon_{q} \epsilon_{2 p}$ are squares in $\mathbb{K}_{2}^{+}$, this gives the F.S.U of $\mathbb{K}_{2}^{+}$, and that of $\mathbb{K}_{2}$ is deduced by Lemma 2.1.

(2) If $N\left(\epsilon_{2 p}\right)=-1$, then $\epsilon_{q} \epsilon_{2 p q}$ is a square in $\mathbb{K}_{2}^{+}$, this gives the F.S.U of $\mathbb{K}_{2}^{+}$, and that of $\mathbb{K}_{2}$ is deduced by Lemma 2.1 .

For the other cases see [1].

3.3. F.S.U of the field $\mathbb{K}_{3}$. Let $\mathbb{K}_{3}=\mathbb{k}_{\mathrm{k}}(\sqrt{2})=\mathbb{Q}(\sqrt{2}, \sqrt{p q}, i)$.

Proposition 3.3. Put $\epsilon_{p q}=a+b \sqrt{p q}$, where $a$ and $b$ are in $\mathbb{Z}$.

(1) If both of $x \pm 1$ and $a \pm 1$ are squares in $\mathbb{N}$, then

(i) If $Q_{\mathbb{K}_{3}}=1$, then $\left\{\epsilon_{2}, \sqrt{\epsilon_{p q}}, \sqrt{\epsilon_{2 p q}}\right\}$ is a F.S.U of both $\mathbb{K}_{3}^{+}$and $\mathbb{K}_{3}$.

(ii) If $Q_{\mathbb{K}_{3}}=2$, then $\left\{\epsilon_{2}, \sqrt{\epsilon_{p q}}, \sqrt{\epsilon_{2 p q}}\right\}$ is a F.S.U of $\mathbb{K}_{3}^{+}$and that of $\mathbb{K}_{3}$ is $\left\{\epsilon_{2}, \sqrt{\epsilon_{p q}}, \sqrt{\xi \sqrt{\epsilon_{p q} \epsilon_{2 p q}}}\right\}$, where $\xi$ is an 8-th root of unity.

(2) If $x \pm 1$ is a square in $\mathbb{N}$ and $a+1, a-1$ are not, then $\left\{\epsilon_{2}, \epsilon_{p q}, \sqrt{\epsilon_{2 p q}}\right\}$ is a F.S.U of both $\mathbb{K}_{3}^{+}$and $\mathbb{K}_{3}$; hence $Q_{\mathbb{K}_{3}}=1$. 
(3) If $a \pm 1$ is a square in $\mathbb{N}$ and $x+1, x-1$ are not, then $\left\{\epsilon_{2}, \epsilon_{2 p q}, \sqrt{\epsilon_{p q}}\right\}$ is a F.S.U of both $\mathbb{K}_{3}^{+}$and $\mathbb{K}_{3}$; hence $Q_{\mathbb{K}_{3}}=1$.

(4) If $x+1, x-1, a+1$ and $a-1$ are not squares in $\mathbb{N}$, then $\left\{\epsilon_{2}, \epsilon_{p q}, \sqrt{\epsilon_{p q} \epsilon_{2 p q}}\right\}$ is a F.S.U of both $\mathbb{K}_{3}^{+}$and $\mathbb{K}_{3}$; hence $Q_{\mathbb{K}_{3}}=1$.

Before proving this proposition, we quote the following result.

Remark 3.4. Keep the notations and hypotheses of the Proposition 3.3 ,

(1) if at most one of the numbers $x+1, x-1, a+1$ and $a-1$ is a square in $\mathbb{N}$, then according to [1, Remarque 13, p. 391$], \mathbb{K}_{3}^{+}$and $\mathbb{K}_{3}$ have the same F.S.U.

(2) From [16, Theorem 2, p. 348 ], if both of $x \pm 1$ and $a \pm 1$ are squares in $\mathbb{N}$, then the unit index of $\mathbb{K}_{3}$ is 1 or 2 .

Proof. We know that $N\left(\epsilon_{2}\right)=-1$ and $N\left(\epsilon_{p q}\right)=N\left(\epsilon_{2 p q}\right)=1$. Moreover, $(2+$ $\sqrt{2}) \epsilon_{2}^{i} \epsilon_{p q}^{j} \epsilon_{2 p q}^{k}$ cannot be a square in $\mathbb{K}_{3}^{+}$, for all $i, j$ and $k$ of $\{0,1\}$; as otherwise with some $\alpha \in \mathbb{K}_{3}^{+}$we would have $\alpha^{2}=(2+\sqrt{2}) \epsilon_{2}^{i} \epsilon_{p q}^{j} \epsilon_{2 p q}^{k}$, so $\left(N_{\mathbb{K}_{3}^{+} / \mathbb{Q}(\sqrt{p q})}(\alpha)\right)^{2}=$ $2(-1)^{i} \epsilon_{p q}^{2 j}$, yielding that $\sqrt{ \pm 2} \in \mathbb{Q}(\sqrt{p q})$, which is absurd.

As $a^{2}-1=p q b^{2}$, so by Lemma 2.2 and according to the decomposition uniqueness in $\mathbb{Z}$, there are three possible cases: $a \pm 1$ or $p(a \pm 1)$ or $2 p(a \pm 1)$ is a square in $\mathbb{N}$.

(a) If $a \pm 1$ is a square in $\mathbb{N}$, then there exist $b_{1}$ and $b_{2}$ in $\mathbb{N}$ with $b=b_{1} b_{2}$ such that $\left\{\begin{array}{l}a \pm 1=b_{1}^{2}, \\ a \mp 1=p q b_{2}^{2},\end{array} \quad\right.$ hence $\sqrt{\epsilon_{p q}}=\frac{1}{2}\left(b_{1} \sqrt{2}+b_{2} \sqrt{2 p q}\right) \in \mathbb{K}_{3}^{+}$.

(b) If $p(a \pm 1)$ is a square in $\mathbb{N}$, then there exist $b_{1}$ and $b_{2}$ in $\mathbb{N}$ with $b=b_{1} b_{2}$ such that $\left\{\begin{array}{l}a \pm 1=p b_{1}^{2}, \\ a \mp 1=q b_{2}^{2},\end{array}\right.$ hence $\left\{\begin{array}{l}\sqrt{\epsilon_{p q}}=\frac{1}{2}\left(b_{1} \sqrt{2 p}+b_{2} \sqrt{2 q}\right) \notin \mathbb{K}_{3}^{+}, \\ \sqrt{p \epsilon_{p q}} \in \mathbb{K}_{3}^{+} \text {and } \sqrt{q \epsilon_{p q}} \in \mathbb{K}_{3}^{+} .\end{array}\right.$

(c) If $2 p(a \pm 1)$ is a square in $\mathbb{N}$, then there exist $b_{1}$ and $b_{2}$ in $\mathbb{N}$ with $b=b_{1} b_{2}$ such that $\left\{\begin{array}{l}a \pm 1=2 p b_{1}^{2}, \\ a \mp 1=2 q b_{2}^{2}\end{array}\right.$ hence $\left\{\begin{array}{l}\sqrt{\epsilon_{p q}}=b_{1} \sqrt{p}+b_{2} \sqrt{q} \notin \mathbb{K}_{3}^{+} ; \\ \sqrt{p \epsilon_{p q}} \in \mathbb{K}_{3}^{+} \text {and } \sqrt{q \epsilon_{p q}} \in \mathbb{K}_{3}^{+} .\end{array}\right.$

Similarly, we get:

(a') If $x \pm 1$ is a square in $\mathbb{N}$, then $\sqrt{\epsilon_{2 p q}} \in \mathbb{K}_{3}^{+}$.

(b') If $p(x \pm 1)$ is a square in $\mathbb{N}$, then $\sqrt{\epsilon_{2 p q}} \notin \mathbb{K}_{3}^{+}, \sqrt{p \epsilon_{2 p q}} \in \mathbb{K}_{3}^{+}$and $\sqrt{q \epsilon_{2 p q}} \in \mathbb{K}_{2}^{+}$.

(c') If $2 p(x \pm 1)$ is a square in $\mathbb{N}$, then $\sqrt{\epsilon_{2 p q}} \notin \mathbb{K}_{3}^{+}, \sqrt{p \epsilon_{2 p q}} \in \mathbb{K}_{2}^{+}$and $\sqrt{q \epsilon_{2 p q}} \in \mathbb{K}_{2}^{+}$.

Consequently, we find:

(1) If $a \pm 1$ and $x \pm 1$ are squares in $\mathbb{N}$, then $\left\{\epsilon_{2}, \sqrt{\epsilon_{p q}}, \sqrt{\epsilon_{2 p q}}\right\}$ is a F.S.U of $\mathbb{K}_{3}^{+}$.

(i) If $Q_{\mathbb{K}_{3}}=1$, then $\left\{\epsilon_{2}, \sqrt{\epsilon_{p q}}, \sqrt{\epsilon_{2 p q}}\right\}$ is also a F.S.U of $\mathbb{K}_{3}$.

(ii) If $Q_{\mathbb{K}_{3}}=2$, then, according to [16], $\mathbb{K}_{3}^{+}(\sqrt{2+\sqrt{2}})=\mathbb{K}_{3}^{+}\left(\sqrt{\sqrt{\epsilon_{p q} \epsilon_{2 p q}}}\right)$, so there exist $\alpha \in \mathbb{K}_{3}^{+}$such that $2+\sqrt{2}=\alpha^{2} \sqrt{\epsilon_{p q} \epsilon_{2 p q}}$. This implies that $(2+\sqrt{2}) \sqrt{\epsilon_{p q} \epsilon_{2 p q}}$ is a square in $\mathbb{K}_{3}^{+}$. Hence Lemma 2.1 yields that $\left\{\epsilon_{2}, \sqrt{\epsilon_{p q}}, \sqrt{\xi \sqrt{\epsilon_{p q} \epsilon_{2 p q}}}\right\}$ is a F.S.U of $\mathbb{K}_{3}$, where $\xi$ is an $8^{\text {th }}$ root of unity.

(2) If $x \pm 1$ is a square in $\mathbb{N}$ and $a+1, a-1$ are not, then $\left\{\epsilon_{2}, \epsilon_{p q}, \sqrt{\epsilon_{2 p q}}\right\}$ is a F.S.U of $\mathbb{K}_{3}^{+}$and, by Remark 3.4, of $\mathbb{K}_{3}$. 
(3) If $a \pm 1$ is a square in $\mathbb{N}$ and $x+1, x-1$ are not, then $\left\{\epsilon_{2}, \epsilon_{2 p q}, \sqrt{\epsilon_{p q}}\right\}$ is a F.S.U of $\mathbb{K}_{3}^{+}$and, by Remark 3.4, of $\mathbb{K}_{3}$.

(4) If $x+1, x-1, a+1$ and $a-1$ are not squares in $\mathbb{N}$, then $\left\{\epsilon_{2}, \epsilon_{p q}, \sqrt{\epsilon_{p q} \epsilon_{2 p q}}\right\}$ is a F.S.U of $\mathbb{K}_{3}^{+}$and, by Remark 3.4, of $\mathbb{K}_{3}$.

\section{The ambiguous classes of $\mathrm{k} / \mathbb{Q}(i)$}

Let $F=\mathbb{Q}(i)$ and $\mathbb{k}=\mathbb{Q}(\sqrt{2 p q}, i)$. We denote by $\mathrm{A} m(\mathbb{k} / F)$ the group of the ambiguous classes of $\mathbb{k} / F$ and by $\mathrm{A}_{s}(\mathrm{k} / F)$ the subgroup of $\mathrm{A} m(\mathrm{k} / F)$ generated by the strongly ambiguous classes. As $p \equiv 1(\bmod 4)$, so there exist $e$ and $f$ in $\mathbb{N}$ such that $p=e^{2}+4 f^{2}=\pi_{1} \pi_{2}$. Put $\pi_{1}=e+2 i f$ and $\pi_{2}=e-2 i f$. Let $\mathcal{H}_{j}$ (resp. $\mathcal{H}_{0}$ ) be the prime ideal of $\mathrm{k}$ above $\pi_{j}$ (resp. $\left.1+i\right), j \in\{1,2\}$. It is easy to see that $\mathcal{H}_{j}^{2}=\left(\pi_{j}\right)$ (resp. $\mathcal{H}_{0}^{2}=(1+i)$ ). Therefore $\left[\mathcal{H}_{j}\right] \in \mathrm{A} m_{s}(\mathrm{k} / F)$, for all $j \in\{0,1,2\}$. Keep the notation $\epsilon_{2 p q}=x+y \sqrt{2 p q}$. In this section, we will determine generators of $\mathrm{A} m_{s}(\mathrm{k} / F)$ and $\mathrm{Am}(\mathbb{k} / F)$. Let us first prove the following result.

Lemma 4.1. Consider the prime ideals $\mathcal{H}_{j}$ of $\mathrm{k}, 0 \leq j \leq 2$.

(1) If $x \pm 1$ is a square in $\mathbb{N}$, then $\left|\left\langle\left[\mathcal{H}_{0}\right],\left[\mathcal{H}_{1}\right],\left[\mathcal{H}_{2}\right]\right\rangle\right|=8$.

(2) Else, $\left[\mathcal{H}_{1}\right]=\left[\mathcal{H}_{2}\right]$ and $\left|\left\langle\left[\mathcal{H}_{0}\right],\left[\mathcal{H}_{1}\right]\right\rangle\right|=4$.

Proof. Since $\mathcal{H}_{0}^{2}=(1+i), \mathcal{H}_{l}^{2}=\left(\pi_{l}\right)$ and $\left(\mathcal{H}_{0} \mathcal{H}_{l}\right)^{2}=\left((1+i) \pi_{l}\right)=((e \mp 2 f)+i(e \pm 2 f))$, where $1 \leq l \leq 2$, and since also $\sqrt{2} \notin \mathbb{Q}(\sqrt{2 p q}), \sqrt{e^{2}+(2 f)^{2}}=\sqrt{p} \notin \mathbb{Q}(\sqrt{2 p q})$ and $\sqrt{(e \mp 2 f)^{2}+(e \pm 2 f)^{2}}=\sqrt{2 p} \notin \mathbb{Q}(\sqrt{2 p q})$, so according to [5. Proposition 1], $\mathcal{H}_{0}, \mathcal{H}_{l}$ and $\mathcal{H}_{0} \mathcal{H}_{l}$ are not principal in $\mathrm{k}$.

(1) If $x \pm 1$ is a square in $\mathbb{N}$, then $p(x+1), p(x-1), 2 p(x+1)$ and $2 p(x-1)$ are not squares in $\mathbb{N}$. Moreover $\left(\mathcal{H}_{1} \mathcal{H}_{2}\right)^{2}=(p)$, hence according to [5, Proposition 2], $\mathcal{H}_{1} \mathcal{H}_{2}$ is not principal in $\mathrm{k}$, and the result derived.

(2) If $x+1$ and $x-1$ are not squares in $\mathbb{N}$, then $p(x \pm 1)$ or $2 p(x \pm 1)$ is a square in $\mathbb{N}$; as $\left(\mathcal{H}_{1} \mathcal{H}_{2}\right)^{2}=(p)$, hence according to [5, Proposition 2], $\mathcal{H}_{1} \mathcal{H}_{2}$ is principal in $\mathrm{k}$. This completes the proof.

Determine now generators of $\mathrm{A} m_{s}(\mathrm{k} / F)$ and $\mathrm{A} m(\mathrm{k} / F)$. According to the ambiguous class number formula (see $[8])$, the genus number, $\left[(\mathbb{k} / F)^{*}: \mathbb{k}\right]$, is given by:

$$
|\mathrm{A} m(\mathbb{k} / F)|=\left[(\mathbb{k} / F)^{*}: \mathbb{k}\right]=\frac{h(F) 2^{t-1}}{\left[E_{F}: E_{F} \cap N_{\mathbb{k} / F}\left(\mathbb{k}^{\times}\right)\right]},
$$

where $h(F)$ is the class number of $F$ and $t$ is the number of finite and infinite primes of $F$ ramified in $\mathrm{k} / F$. Moreover as the class number of $F$ is equal to 1 , so the formula (11) yields that

$$
|\mathrm{A} m(\mathbb{k} / F)|=\left[(\mathbb{k} / F)^{*}: \mathbb{k}\right]=2^{r},
$$

where $r=\operatorname{rank} \mathbf{C} l_{2}(\mathbb{k})=t-e-1$ and $2^{e}=\left[E_{F}: E_{F} \cap N_{\mathbb{k} / F}\left(\mathbb{k}^{\times}\right)\right]$(see for example [21]). The relation between $|\mathrm{A} m(\mathbb{k} / F)|$ and $\left|\mathrm{A} m_{s}(\mathbb{k} / F)\right|$ is given by the following 
formula (see for example [9]):

$$
\frac{|\mathrm{A} m(\mathbb{k} / F)|}{\left|\mathrm{A} m_{s}(\mathbb{k} / F)\right|}=\left[E_{F} \cap N_{\mathbb{k} / F}\left(\mathbb{k}^{\times}\right): N_{\mathbb{k} / F}\left(E_{\mathbb{k}}\right)\right] .
$$

To continue, we need the following lemma.

Lemma 4.2. Let $p \equiv-q \equiv 1(\bmod 4)$ be different primes, $F=\mathbb{Q}(i)$ and $\mathbb{k}=$ $\mathbb{Q}(\sqrt{2 p q}, i)$.

(1) If $p \equiv 1(\bmod 8)$, then $i$ is a norm in $\mathrm{k} / F$.

(2) If $p \equiv 5(\bmod 8)$, then $i$ is not a norm in $\mathrm{k} / F$.

Proof. Let $\mathfrak{p}$ be a prime ideal of $F=\mathbb{Q}(i)$ such that $\mathfrak{p} \neq 2_{F}$, where $2_{F}$ is the prime ideal of $F$ above 2 , then the Hilbert symbol yields that $\left(\frac{2 p q, i}{\mathfrak{p}}\right)=\left(\frac{p q, i}{\mathfrak{p}}\right)$, since $2 i=(1+i)^{2}$. Hence, by Hilbert symbol properties and according to [12, p. 205], we get:

- If $\mathfrak{p}$ is not above $p$ and $q$, then $v_{\mathfrak{p}}(p q)=0$, thus $\left(\frac{p q, i}{\mathfrak{p}}\right)=1$.

- If $\mathfrak{p}$ lies above $p$, then $v_{\mathfrak{p}}(p q)=1$, so

$$
\left(\frac{p q, i}{\mathfrak{p}}\right)=\left(\frac{i}{\mathfrak{p}}\right)=\left(\frac{2}{p}\right) \text {, indeed }\left(\frac{2}{p}\right)\left(\frac{i}{\mathfrak{p}}\right)=\left(\frac{2}{\mathfrak{p}}\right)\left(\frac{i}{\mathfrak{p}}\right)=\left(\frac{2 i}{\mathfrak{p}}\right)=1 \text {. }
$$

- If $\mathfrak{p}$ lies above $q$, then $v_{\mathfrak{p}}(p q)=1$, so

$$
\begin{aligned}
& \left(\frac{p q, i}{\mathfrak{p}}\right)=\left(\frac{i}{\mathfrak{p}}\right)=\left(\frac{N_{F / \mathbb{Q}}(i)}{q}\right)=\left(\frac{1}{q}\right)=1 \text {, since } q \text { remained inert in } \\
& F / \mathbb{Q} \text {. }
\end{aligned}
$$

So for every prime ideal $\mathfrak{p} \in F$ and by the product formula for the Hilbert symbol, we deduce that $\left(\frac{p q, i}{\mathfrak{p}}\right)=1$, hence:

(1) If $p \equiv 1(\bmod 8)$, then $i$ is a norm in $\mathrm{k} / F$.

(2) If $p \equiv 5(\bmod 8)$, then $i$ is not a norm in $\mathrm{k} / F$.

Proposition 4.3. Let $(\mathbb{k} / F)^{*}$ denote the relative genus field of $\mathbb{k} / F$.

(1) $\mathbb{k}^{(*)} \subseteq(\mathbb{k} / F)^{*}$ and $\left[(\mathbb{k} / F)^{*}: \mathbb{k}^{(*)}\right] \leq 2$.

(2) Assume $p \equiv 1(\bmod 8)$.

(i) If $x \pm 1$ is a square in $\mathbb{N}$, then $\operatorname{Am}(\mathbb{k} / \mathbb{Q}(i))=\operatorname{Am}_{s}(\mathbb{k} / \mathbb{Q}(i))=$ $\left\langle\left[\mathcal{H}_{0}\right],\left[\mathcal{H}_{1}\right],\left[\mathcal{H}_{2}\right]\right\rangle$.

(ii) Else, there exist an unambiguous ideal $\mathcal{I}$ in $\mathbb{k} / \mathbb{Q}(i)$ of order 2 such that $\operatorname{Am}_{s}(\mathbb{k} / \mathbb{Q}(i))=\left\langle\left[\mathcal{H}_{0}\right],\left[\mathcal{H}_{1}\right]\right\rangle$ and $\operatorname{Am}(\mathbb{k} / \mathbb{Q}(i))=\left\langle\left[\mathcal{H}_{0}\right],\left[\mathcal{H}_{1}\right],[\mathcal{I}]\right\rangle$.

(3) Assume $p \equiv 5(\bmod 8)$, then neither $x+1$ nor $x-1$ is a square in $\mathbb{N}$ and $\operatorname{Am}(\mathbb{k} / \mathbb{Q}(i))=\operatorname{Am}_{s}(\mathbb{k} / \mathbb{Q}(i))=\left\langle\left[\mathcal{H}_{0}\right],\left[\mathcal{H}_{1}\right]\right\rangle$.

Proof. (1) As $\mathbb{k}=\mathbb{Q}(\sqrt{2 p q}, i)$, so $\left[\mathbb{k}^{(*)}: \mathbb{k}\right]=4$. Moreover, according to [21, Proposition 2, p. 90], $r=\operatorname{rank} \mathbf{C} l_{2}(\mathbb{k})=3$ if $p \equiv 1(\bmod 8)$ and $r=\operatorname{rank} \mathbf{C} l_{2}(\mathbb{k})=$ 2 if $p \equiv 5(\bmod 8)$, so $\left[(\mathbb{k} / F)^{*}: \mathbb{k}\right]=4$ or 8 . Hence $\left[(\mathbb{k} / F)^{*}: \mathbb{k}^{(*)}\right] \leq 2$, and the result derived. 
(2) Assume that $p \equiv 1(\bmod 8)$, hence $i$ is a norm in $\mathbb{k} / \mathbb{Q}(i)$, thus Formula (3) yields that

$$
\begin{aligned}
\frac{|\operatorname{Am}(\mathbb{k} / \mathbb{Q}(i))|}{\left|\operatorname{Am}_{s}(\mathbb{k} / \mathbb{Q}(i))\right|} & =\left[E_{\mathbb{Q}(i)} \cap N_{\mathbb{k} / \mathbb{Q}(i)}\left(\mathbb{k}^{\times}\right): N_{\mathbb{k} / \mathbb{Q}(i)}\left(E_{\mathbb{k}}\right)\right] \\
& =\left\{\begin{array}{l}
1 \text { if } x \pm 1 \text { is a square in } \mathbb{N}, \\
2 \text { if not, }
\end{array}\right.
\end{aligned}
$$

since in the case where $x \pm 1$ is a square in $\mathbb{N}$, we have $E_{\mathbb{k}}=\left\langle i, \sqrt{i \epsilon_{2 p q}}\right\rangle$, hence $\left[E_{\mathbb{Q}(i)} \cap N_{\mathbb{k} / \mathbb{Q}(i)}\left(\mathbb{k}^{\times}\right): N_{\mathbb{k} / \mathbb{Q}(i)}\left(E_{\mathbb{k}}\right)\right]=[\langle i>:\langle i>]=1$, and if not we have $E_{\mathbb{k}}=\left\langle i, \epsilon_{2 p q}\right\rangle$, hence $\left.\left[E_{\mathbb{Q}(i)} \cap N_{\mathbb{k} / \mathbb{Q}(i)}\left(\mathbb{k}^{\times}\right): N_{\mathbb{k} / \mathbb{Q}(i)}\left(E_{\mathbb{k}}\right)\right]=[\langle i\rangle:<-1\rangle\right]=2$.

On the other hand, as $p \equiv 1(\bmod 8)$, so according to [21, Proposition 2, p. 90], $r=3$. Therefore $|\operatorname{Am}(\mathbb{k} / \mathbb{Q}(i))|=2^{3}$.

(i) If $x \pm 1$ is a square in $\mathbb{N}$, then $\operatorname{Am}_{s}(\mathbb{k} / \mathbb{Q}(i))=\operatorname{Am}(\mathbb{k} / \mathbb{Q}(i))$, hence by Lemma 4.1 we get $\operatorname{Am}(\mathbb{k} / \mathbb{Q}(i))=\operatorname{Am}_{s}(\mathbb{k} / \mathbb{Q}(i))=\left\langle\left[\mathcal{H}_{0}\right],\left[\mathcal{H}_{1}\right],\left[\mathcal{H}_{2}\right]\right\rangle$.

(ii) If $x+1$ and $x-1$ are not squares in $\mathbb{N}$, then

$$
|\operatorname{Am}(\mathbb{k} / \mathbb{Q}(i))|=2\left|\operatorname{Am}_{s}(\mathbb{k} / \mathbb{Q}(i))\right|=8,
$$

hence Lemma 4.1]yields that $\operatorname{Am}_{s}(\mathbb{k} / \mathbb{Q}(i))=\left\langle\left[\mathcal{H}_{0}\right],\left[\mathcal{H}_{1}\right]\right\rangle$.

Consequently, there exist an unambiguous ideal $\mathcal{I}$ in $\mathrm{k} / F$ of order 2 such that

$$
\operatorname{Am}(\mathbb{k} / \mathbb{Q}(i))=\left\langle\left[\mathcal{H}_{0}\right],\left[\mathcal{H}_{1}\right],[\mathcal{I}]\right\rangle .
$$

By Chebotarev theorem, $\mathcal{I}$ can always be chosen as a prime ideal of $\mathbb{k}$ above a prime $l$ in $\mathbb{Q}$, which splits completely in $\mathbb{k}$. So we can determine $\mathcal{I}$ by using the following lemma.

Lemma 4.4 ([17]). Let $p_{1}, p_{2}, \ldots, p_{n}$ be distinct primes and for each $j$, let $e_{j}= \pm 1$. Then there exist infinitely many primes $l$ such that $\left(\frac{p_{j}}{l}\right)=e_{j}$, for all $j$.

Let $l \equiv 1(\bmod 4)$ be a prime satisfying $\left(\frac{2 p q}{l}\right)=-\left(\frac{q}{l}\right)=1$, then $l$ splits completely in $\mathbb{k}$. Let $\mathcal{I}$ be a prime ideal of $\mathbb{k}$ above $l$; hence $\mathcal{I}$ remained inert in $\mathbb{K}_{2}$ and $\left(\frac{2 p}{l}\right)=-1$. We need to prove that $\mathcal{I}, \mathcal{H}_{0} \mathcal{I}, \mathcal{H}_{1} \mathcal{I}$ and $\mathcal{H}_{0} \mathcal{H}_{1} \mathcal{I}$ are not principal in $\mathrm{k}$.

- As $\mathcal{I}$ remained inert in $\mathbb{K}_{2}$, so $\varphi_{\mathbb{K}_{2} / \mathbb{k}}(\mathcal{I}) \neq 1$, where $\varphi_{\mathbb{K}_{2} / \mathbb{k}}$ denotes the Artin map of $\mathbb{K}_{2}$ over $\mathbb{k}$, similarly, we have $\varphi_{\mathbb{K}_{2} / \mathbb{k}}\left(\mathcal{H}_{1} \mathcal{I}\right) \neq 1$ (note that $\left(\frac{p}{q}\right)=1$, since $p(x \pm 1)$ or $2 p(x \pm 1)$ is a square in $\mathbb{N})$. Therefore $\mathcal{I}$ and $\mathcal{H}_{1} \mathcal{I}$ are not principal in $\mathrm{k}$.

- Let us prove that $\mathcal{H}_{0} \mathcal{I}$ is not principal in $\mathrm{k}$. For this, we consider the following cases:

(a) Assume $\left(\frac{2}{l}\right)=1$, then $\left(\frac{p}{l}\right)=-1$; thus if $\left(\frac{2}{q}\right)=-1$, then $\varphi_{\mathbb{K}_{3} / \mathbb{k}}\left(\mathcal{H}_{0} \mathcal{I}\right) \neq 1$, and if $\left(\frac{2}{q}\right)=1$, then $\varphi_{\mathbb{K}_{1} / \mathbb{k}}\left(\mathcal{H}_{0} \mathcal{I}\right) \neq 1$. Hence $\mathcal{H}_{0} \mathcal{I}$ is not principal in $\mathbb{k}$. 
(b) Assume now $\left(\frac{2}{l}\right)=-1$, hence $\left(\frac{p}{l}\right)=1$. Thus if $\left(\frac{2}{q}\right)=1$, then $\varphi_{\mathbb{K}_{2} / \mathbb{k}}\left(\mathcal{H}_{0} \mathcal{I}\right) \neq$ 1. If $\left(\frac{2}{q}\right)=-1$, so we need the following two quadratic extensions of $\mathbb{k}$ : $\mathbb{K}_{4}=\mathbb{k}\left(\sqrt{\pi_{1}}\right)$ and $\mathbb{K}_{5}=\mathbb{k}_{k}\left(\sqrt{2 \pi_{1}}\right)=\mathbb{k}\left(\sqrt{\pi_{2} q}\right)$, where $p=e^{2}+16 f^{2}=$ $\pi_{1} \pi_{2}=(e+4 i f)(e-4 i f)$, since $p \equiv 1(\bmod 8)$. Note that $\mathbb{K}_{4} / \mathbb{k}_{\mathrm{k}}$ and $\mathbb{K}_{5} / \mathbb{k}_{\mathrm{k}}$ are unramified (see [4]). As $\left(\frac{2}{p}\right)=1$, then $\left(\frac{1+i}{\pi_{1}}\right)=\left(\frac{1+i}{\pi_{2}}\right)$, hence the quadratic residue symbol implies that

$$
\left(\frac{\pi_{1}}{\mathcal{H}_{0} \mathcal{I}}\right)=\left(\frac{1+i}{\pi_{1}}\right)=-\left(\frac{\pi_{2} q}{\mathcal{H}_{0} \mathcal{I}}\right)
$$

Therefore, if $\left(\frac{1+i}{\pi_{1}}\right)=-1$, then $\varphi_{\mathbb{K}_{4} / \mathbb{k}}\left(\mathcal{H}_{0} \mathcal{I}\right) \neq 1$, else we have $\varphi_{\mathbb{K}_{5} / \mathbb{k}}\left(\mathcal{H}_{0} \mathcal{I}\right) \neq$ 1. Thus $\mathcal{H}_{0} \mathcal{I}$ is not principal in $\mathbb{k}$.

With the same argument, we show that $\mathcal{H}_{0} \mathcal{H}_{1} \mathcal{I}$ is not principal in $\mathbb{k}$.

(3) Assume that $p \equiv 5(\bmod 8)$, hence $i$ is not a norm in $\mathrm{k} / \mathbb{Q}(i)$ and $x+1$, $x-1$ are not squares in $\mathbb{N}$, for if $x \pm 1$ is a square in $\mathbb{N}$, then the Legendre symbol implies that

$$
1=\left(\frac{x \pm 1}{p}\right)=\left(\frac{x \mp 1 \pm 2}{p}\right)=\left(\frac{2}{p}\right),
$$

which is absurd. Thus $|\operatorname{Am}(\mathbb{k} / \mathbb{Q}(i))|=2^{2}$ and

$$
\frac{|\operatorname{Am}(\mathbb{k} / \mathbb{Q}(i))|}{\left|\operatorname{Am}_{s}(\mathbb{k} / \mathbb{Q}(i))\right|}=\left[E_{\mathbb{Q}(i)} \cap N_{\mathbb{k} / \mathbb{Q}(i)}\left(\mathbb{k}^{\times}\right): N_{\mathbb{k} / \mathbb{Q}(i)}\left(E_{\mathbb{k}}\right)\right]=1 .
$$

Hence by Lemma 4.1 we get $\operatorname{Am}(\mathbb{k} / \mathbb{Q}(i))=\operatorname{Am}_{s}(\mathbb{k} / \mathbb{Q}(i))=\left\langle\left[\mathcal{H}_{0}\right],\left[\mathcal{H}_{1}\right]\right\rangle$. This completes the proof.

\section{Capitulation}

In this section, we will determine the classes of $\mathbf{C} l_{2}(\mathbb{k})$, the 2-class group of $\mathbb{k}$, that capitulate in $\mathbb{K}_{j}$, for all $j \in\{1,2,3\}$. For this we need the following theorem.

Theorem 5.1 ([10]). Let $K / k$ be a cyclic extension of prime degree, then the number of classes that capitulate in $K / k$ is: $[K: k]\left[E_{k}: N_{K / k}\left(E_{K}\right)\right]$, where $E_{k}$ and $E_{K}$ are the unit groups of $k$ and $K$ respectively.

Theorem 5.2. Let $\mathbb{K}_{j}, 1 \leq j \leq 3$, be the three unramified quadratic extensions of $\mathrm{k}$ defined above.

(1) For $j \in\{1,2\}$ we have

(i) If $x \pm 1$ is a square in $\mathbb{N}$, then $\left|\kappa_{\mathbb{K}_{j}}\right|=4$.

(ii) Else $\left|\kappa_{\mathbb{K}_{j}}\right|=2$.

(2) Put $\epsilon_{p q}=a+b \sqrt{p q}$ and let $Q_{\mathbb{K}_{3}}$ denote the unit index of $\mathbb{K}_{3}$.

(i) If both of $x \pm 1$ and $a \pm 1$ are squares in $\mathbb{N}$, then

(a) If $Q_{\mathbb{K}_{3}}=1$, then $\left|\kappa_{\mathbb{K}_{3}}\right|=4$.

(b) If $Q_{\mathbb{K}_{3}}=2$, then $\left|\kappa_{\mathbb{K}_{3}}\right|=2$. 
(ii) If one of the four numbers $x+1, x-1, a+1$ and $a-1$ is a square in $\mathbb{N}$, and the others are not, then $\left|\kappa_{\mathbb{K}_{3}}\right|=4$.

(iii) If $x+1, x-1, a+1$ and $a-1$ are not squares in $\mathbb{N}$, then $\left|\kappa_{\mathbb{K}_{3}}\right|=2$.

Proof. (1) According to Proposition 3.1, $E_{\mathbb{K}_{1}}=\left\langle i, \epsilon_{p}, \sqrt{i \epsilon_{2 q}}, \sqrt{\epsilon_{2 q} \epsilon_{2 p q}}\right\rangle$ or $\left\langle i, \epsilon_{p}, \sqrt{i \epsilon_{2 q}}, \sqrt{\epsilon_{2 p q}}\right\rangle$, so $N_{\mathbb{K}_{1} / \mathbb{k}}\left(E_{\mathbb{K}_{1}}\right)=\left\langle i, \epsilon_{2 p q}\right\rangle$. On the other hand, Proposition 3.2 yields that $E_{\mathbb{K}_{2}}=\left\langle i, \sqrt{i \epsilon_{q}}, \sqrt{i \epsilon_{2 p}}, \sqrt{i \epsilon_{2 p q}}\right\rangle$ or $\left\langle i, \sqrt{i \epsilon_{q}}, \sqrt{i \epsilon_{2 p}}, \sqrt{\epsilon_{2 p q}}\right\rangle$ or $\left\langle i, \sqrt{i \epsilon_{q}}, \epsilon_{2 p}, \sqrt{\epsilon_{q} \epsilon_{2 p q}}\right\rangle$ or $\left\langle i, \sqrt{i \epsilon_{q}}, \epsilon_{2 p}, \sqrt{\epsilon_{2 p q}}\right\rangle$, hence $N_{\mathbb{K}_{2} / \mathbb{k}_{\mathrm{k}}}\left(E_{\mathbb{K}_{2}}\right)=\left\langle i, \epsilon_{2 p q}\right\rangle$.

(i) If $x \pm 1$ is a square in $\mathbb{N}$, then Lemma 2.5 yields that $E_{\mathbb{k}}=\left\langle i, \sqrt{i \epsilon_{2 p q}}\right\rangle$. Therefore $\left[E_{\mathbb{k}}: N_{\mathbb{K}_{j} / \mathbb{k}_{\mathrm{k}}}\left(E_{\mathbb{K}_{j}}\right)\right]=2$, and Theorem 5.1 implies that $\left|\kappa_{\mathbb{K}_{j}}\right|=4$.

(ii) Else $E_{\mathbb{k}}=\left\langle i, \epsilon_{2 p q}\right\rangle$, which gives that $\left[E_{\mathbb{K}_{\mathrm{k}}}: N_{\mathbb{K}_{j} / \mathbb{k}_{\mathrm{k}}}\left(E_{\mathbb{K}_{j}}\right)\right]=1$, and Theorem 5.1 implies that $\left|\kappa_{\mathbb{K}_{1}}\right|=2$.

(2) (i) Assume that $x \pm 1$ and $a \pm 1$ are squares in $\mathbb{N}$, so by Lemma 2.5 we get $E_{\mathrm{k}}=\left\langle i, \sqrt{i \epsilon_{2 p q}}\right\rangle$.

(a) If $Q_{\mathbb{K}_{3}}=1$, then Proposition 3.3 implies that $E_{\mathbb{K}_{3}}=\left\langle\sqrt{i}, \epsilon_{2}, \sqrt{\epsilon_{p q}}, \sqrt{\epsilon_{2 p q}}\right\rangle$, hence $N_{\mathbb{K}_{3} / \mathbb{k}}\left(E_{\mathbb{K}_{3}}\right)=\left\langle i, \epsilon_{2 p q}\right\rangle$, from which we deduce that $\left[E_{\mathbb{k}}: N_{\mathbb{K}_{3} / \mathbb{k}_{\mathrm{k}}}\left(E_{\mathbb{K}_{3}}\right)\right]=$ 2 , and Theorem 5.1 implies that $\left|\kappa_{\mathbb{K}_{3}}\right|=4$.

(b) If $Q_{\mathbb{K}_{3}}=2$, then Proposition 3.3 implies that $E_{\mathbb{K}_{3}}=\left\langle\sqrt{i}, \epsilon_{2}, \sqrt{\epsilon_{p q}}, \sqrt{\sqrt{i \epsilon_{p q} \epsilon_{2 p q}}}\right\rangle$, thus $N_{\mathbb{K}_{3} / \mathbb{k}}\left(E_{\mathbb{K}_{3}}\right)=\left\langle i, \sqrt{i \epsilon_{2 p q}}\right\rangle$, from which we deduce that $\left[E_{\mathbb{k}}: N_{\mathbb{K}_{3} / \mathbb{k}}\left(E_{\mathbb{K}_{3}}\right)\right]=$ 1 , and Theorem 5.1 implies that $\left|\kappa_{\mathbb{K}_{3}}\right|=2$.

(ii) If $x \pm 1$ is a square in $\mathbb{N}$ and $a+1, a-1$ are not, then by Lemma 2.5 we get $E_{\mathbb{k}}=\left\langle i, \sqrt{i \epsilon_{2 p q}}\right\rangle$. Moreover, Proposition 3.3 implies that $E_{\mathbb{K}_{3}}=$ $\left\langle\sqrt{i}, \epsilon_{2}, \epsilon_{p q}, \sqrt{\epsilon_{2 p q}}\right\rangle$, hence $N_{\mathbb{K}_{3} / \mathbb{k}_{\mathrm{k}}}\left(E_{\mathbb{K}_{3}}\right)=\left\langle i, \epsilon_{2 p q}\right\rangle$. Therefore $\left[E_{\mathbb{k}}: N_{\mathbb{K}_{3} / \mathbb{k}_{\mathrm{k}}}\left(E_{\mathbb{K}_{3}}\right)\right]=$ 2 , and Theorem 5.1 implies that $\left|\kappa_{\mathbb{K}_{3}}\right|=4$.

If $a \pm 1$ is a square in $\mathbb{N}$ and $x+1, x-1$ are not, then by Lemma 2.5 we get $E_{\mathbb{k}}=\left\langle i, \epsilon_{2 p q}\right\rangle$. Moreover Proposition 3.3 implies that $E_{\mathbb{K}_{3}}=\left\langle\sqrt{i}, \epsilon_{2}, \sqrt{\epsilon_{p q}}, \epsilon_{2 p q}\right\rangle$, hence $N_{\mathbb{K}_{3} / \mathbb{k}}\left(E_{\mathbb{K}_{3}}\right)=\left\langle i, \epsilon_{2 p q}^{2}\right\rangle$. Therefore $\left[E_{\mathbb{k}}: N_{\mathbb{K}_{3} / \mathbb{k}}\left(E_{\mathbb{K}_{3}}\right)\right]=2$, and Theorem 5.1 implies that $\left|\kappa_{\mathbb{K}_{3}}\right|=4$.

(iii) Finally, assume that $x+1, x-1, a+1$ and $a-1$ are not squares in $\mathbb{N}$, then by Lemma 2.5 we get $E_{\mathrm{lk}}=\left\langle i, \epsilon_{2 p q}\right\rangle$. Moreover, Proposition $3.3 \mathrm{im}$ plies that $E_{\mathbb{K}_{3}}=\left\langle\sqrt{i}, \epsilon_{2}, \epsilon_{p q}, \sqrt{\epsilon_{p q} \epsilon_{2 p q}}\right\rangle$, hence $N_{\mathbb{K}_{3} / \mathbb{k}}\left(E_{\mathbb{K}_{3}}\right)=\left\langle i, \epsilon_{2 p q}\right\rangle$. Therefore $\left[E_{\mathbb{k}}: N_{\mathbb{K}_{3} / \mathbb{k}}\left(E_{\mathbb{K}_{3}}\right)\right]=1$, and Theorem 5.1 implies that $\left|\kappa_{\mathbb{K}_{3}}\right|=2$.

\subsection{Capitulation in $\mathbb{K}_{1}$.}

Theorem 5.3. Keep the notations and hypotheses previously mentioned.

(1) If $x \pm 1$ is a square in $\mathbb{N}$, then $\kappa_{\mathbb{K}_{1}}=\left\langle\left[\mathcal{H}_{1}\right],\left[\mathcal{H}_{2}\right]\right\rangle$.

(2) Else $\kappa_{\mathbb{K}_{1}}=\left\langle\left[\mathcal{H}_{1}\right]\right\rangle$.

Proof. Let us first prove that $\mathcal{H}_{1}$ and $\mathcal{H}_{2}$ capitulate in $\mathbb{K}_{1}$. As $N\left(\epsilon_{p}\right)=-1$, then $s^{2}+4=t^{2} p$, where $\epsilon_{p}=\frac{1}{2}(s+t \sqrt{p})$, hence $(s-2 i)(s+2 i)=t^{2} p$. According 
to the decomposition uniqueness in $\mathbb{Z}[i]$, there exist $t_{1}$ and $t_{2}$ in $\mathbb{Z}[i]$ such that: (1) $\left\{\begin{array}{l}s \pm 2 i=t_{1}^{2} \pi_{1} \\ s \mp 2 i=t_{2}^{2} \pi_{2},\end{array}\right.$ or (2) $\left\{\begin{array}{l}s \pm 2 i=i t_{1}^{2} \pi_{1} \\ s \mp 2 i=-i t_{2}^{2} \pi_{2},\end{array}\right.$ where $t=t_{1} t_{2}$.

- The system (1) implies that $2 s=t_{1}^{2} \pi_{1}+t_{2}^{2} \pi_{2}$. Put $\alpha=\frac{1}{2}\left(t_{1} \pi_{1}+t_{2} \sqrt{p}\right)$ and $\beta=\frac{1}{2}\left(t_{2} \pi_{2}+t_{1} \sqrt{p}\right)$. Then $\alpha$ and $\beta$ are in $\mathbb{K}_{1}=\mathbb{k}(\sqrt{p})$ and we have:

$$
\begin{aligned}
\alpha^{2} & =\frac{1}{4}\left(t_{1}^{2} \pi_{1}^{2}+t_{2}^{2} p+2 t_{1} t_{2} \pi_{1} \sqrt{p}\right) \\
& =\frac{1}{4} \pi_{1}\left(t_{1}^{2} \pi_{1}+t_{2}^{2} \pi_{2}+2 t \sqrt{p}\right), \quad \text { since } p=\pi_{1} \pi_{2} \text { and } t=t_{1} t_{2} . \\
& =\frac{1}{4} \pi_{1}(2 s+2 t \sqrt{p}), \quad \text { since } 2 s=t_{1}^{2} \pi_{1}+t_{2}^{2} \pi_{2} . \\
& =\pi_{1} \epsilon_{p}, \quad \text { since } \epsilon_{p}=\frac{1}{2}(s+t \sqrt{p}) .
\end{aligned}
$$

The same argument yields that $\beta^{2}=\pi_{2} \epsilon_{p}$.

Consequently, $\left(\alpha^{2}\right)=\left(\pi_{1}\right)=\mathcal{H}_{1}^{2}\left(\operatorname{resp} .\left(\beta^{2}\right)=\left(\pi_{2}\right)=\mathcal{H}_{2}^{2}\right)$, hence $(\alpha)=\mathcal{H}_{1}$ and $(\beta)=\mathcal{H}_{2}$.

- Similarly, system $(2)$ yields that $2 s=i t_{1}^{2} \pi_{2}-i t_{2}^{2} \pi_{1}$, hence $\sqrt{2 \pi_{1} \epsilon_{p}}=\frac{1}{2}\left(t_{1}(1+\right.$ i) $\left.\pi_{1}+t_{2}(1-i) \sqrt{p}\right)$ and $\sqrt{2 \pi_{2} \epsilon_{p}}=\frac{1}{2}\left(t_{1}(1+i) \sqrt{p}+t_{2}(1-i) \pi_{2}\right)$ are in $\mathbb{K}_{1}$. Therefore there exist $\alpha$ and $\beta$ in $\mathbb{K}_{1}$ such that $2 \pi_{1} \epsilon_{p}=\alpha^{2}$ and $2 \pi_{2} \epsilon_{p}=\beta^{2}$, thus $\left(\frac{\alpha}{1+i}\right)=\mathcal{H}_{1}$ and $\left(\frac{\beta}{1+i}\right)=\mathcal{H}_{2}$. This yields that $\mathcal{H}_{1}$ and $\mathcal{H}_{2}$ capitulate in $\mathbb{K}_{1}$.

On the other hand, by Lemma 4.1. $\mathcal{H}_{j}, 1 \leq j \leq 2$, are not principal in $\mathbb{k}$.

(1) If $x \pm 1$ is a square in $\mathbb{N}$, then Lemma 4.1 yields that $\left[\mathcal{H}_{1} \mathcal{H}_{2}\right] \neq 1$. Hence the result.

(2) If $x+1$ and $x-1$ are not squares in $\mathbb{N}$, then Lemma 4.1 yields that $\left[\mathcal{H}_{1}\right]=\left[\mathcal{H}_{2}\right]$. This completes the proof.

5.2. Capitulation in $\mathbb{K}_{2}$. We need the following two lemmas.

Lemma 5.4. If $N\left(\epsilon_{2 p}\right)=1$, then

(1) $p \equiv 1(\bmod 8)$.

(2) $2 p(x-1)$ is not a square in $\mathbb{N}$.

Proof. (1) Put $\epsilon_{2 p}=\alpha+\beta \sqrt{2 p}$, then, if $N\left(\epsilon_{2 p}\right)=1$, Lemma 2.4 yields that $\left\{\begin{array}{l}\alpha \pm 1=\beta_{1}^{2}, \\ \alpha \mp 1=2 p \beta_{2}^{2},\end{array}\right.$ hence $1=\left(\frac{\alpha \pm 1}{p}\right)=\left(\frac{\alpha \mp 1 \pm 2}{p}\right)=\left(\frac{2}{p}\right)$, so the result.

(2) If $2 p(x-1)$ is a square in $\mathbb{N}$, then $\left\{\begin{array}{l}x-1=2 p y_{1}^{2} \\ x+1=q y_{2}^{2}\end{array}\right.$ thus

$$
\left\{\begin{array}{l}
\left(\frac{2 p}{q}\right)=\left(\frac{x-1}{q}\right)=-\left(\frac{2}{q}\right), \\
\left(\frac{q}{p}\right)=\left(\frac{x+1}{p}\right)=\left(\frac{2}{p}\right)
\end{array}\right.
$$

this implies that $\left(\frac{2}{p}\right)=-1$, which contradicts the first assertion (1). 
Lemma 5.5. Put $\epsilon_{p q}=a+b \sqrt{p q}$. If $a \pm 1$ is a square in $\mathbb{N}$, then $p \equiv 1(\bmod 8)$.

Proof. The same argument as in Lemma 5.4(1) leads to the result.

Theorem 5.6. Keep the notations and hypotheses previously mentioned.

(1) If $N\left(\epsilon_{2 p}\right)=1$ and $x \pm 1$ is a square in $\mathbb{N}$, then $\kappa_{\mathbb{K}_{2}}=\left\langle\left[\mathcal{H}_{0}\right],\left[\mathcal{H}_{1} \mathcal{H}_{2}\right]\right\rangle$ or $\left\langle\left[\mathcal{H}_{1}\right],\left[\mathcal{H}_{2}\right]\right\rangle$.

(2) If $N\left(\epsilon_{2 p}\right)=1$ and $x+1, x-1$ are not squares in $\mathbb{N}$, then there exist an unambiguous ideal $\mathcal{I}$ in $\mathbb{k} / F$ of order 2 , such that $\kappa_{\mathbb{K}_{2}}=\langle[\mathcal{I}]\rangle$ or $\left\langle\left[\mathcal{H}_{0} \mathcal{I}\right]\right\rangle$ or $\left\langle\left[\mathcal{H}_{1} \mathcal{I}\right]\right\rangle$ or $\left\langle\left[\mathcal{H}_{0} \mathcal{H}_{1} \mathcal{I}\right]\right\rangle$.

(3) If $N\left(\epsilon_{2 p}\right)=-1$, then

(i) If $x \pm 1$ is a square in $\mathbb{N}$, then $\kappa_{\mathbb{K}_{2}}=\left\langle\left[\mathcal{H}_{0} \mathcal{H}_{1}\right],\left[\mathcal{H}_{0} \mathcal{H}_{2}\right]\right\rangle$

(ii) Else, $\kappa_{\mathbb{K}_{2}}=\left\langle\left[\mathcal{H}_{0} \mathcal{H}_{1}\right]\right\rangle$.

Proof. Since $\left(\pi_{j}\right)=\mathcal{H}_{j}^{2}, j \in\{1,2\}$, and $\mathcal{H}_{0}^{2}=(1+i)$, so $(2 p)=\left((1+i) \mathcal{H}_{1} \mathcal{H}_{2}\right)^{2}$. Moreover, $2 p$ is a square in $\mathbb{K}_{2}$, so there exist $\alpha \in \mathbb{K}_{2}$ such that $(2 p)=\left(\alpha^{2}\right)$, hence $\left((1+i) \mathcal{H}_{1} \mathcal{H}_{2}\right)^{2}=\left(\alpha^{2}\right)$, therefore $\mathcal{H}_{1} \mathcal{H}_{2}=\left(\frac{\alpha}{1+i}\right)$ and $\mathcal{H}_{1} \mathcal{H}_{2}$ capitulates in $\mathbb{K}_{2}$.

(1) If $N\left(\epsilon_{2 p}\right)=1$, then by Lemma 5.4 we get $p \equiv 1(\bmod 8)$. Moreover, according to Lemma 4.1, if $x \pm 1$ is a square in $\mathbb{N}$, then $\mathcal{H}_{1}, \mathcal{H}_{2}$ and $\mathcal{H}_{1} \mathcal{H}_{2}$ are not principal in $\mathbb{k}$ and, according to the Theorem 5.2 , there are four classes that capitulate in $\mathbb{K}_{2}$. The following examples affirm the two cases of capitulation:

\begin{tabular}{|l|l|l|l|l|l|l|l|}
\hline$d=2 p q$ & $x+1$ & $\mathcal{H}_{0} \mathcal{O}_{\mathbb{K}_{2}}$ & $\mathcal{H}_{1} \mathcal{O}_{\mathbb{K}_{2}}$ & $\mathcal{H}_{2} \mathcal{O}_{\mathbb{K}_{2}}$ & $\mathcal{H}_{1} \mathcal{H}_{2} \mathcal{O}_{\mathbb{K}_{2}}$ & $\mathbf{C l}(\mathbb{k})$ & $\mathbf{C l}\left(\mathbb{K}_{2}\right)$ \\
\hline $238=2.17 .7$ & $108^{2}$ & {$[0,0,0]$} & {$[4,0,0]$} & {$[4,0,0]$} & {$[0,0,0]$} & $(4,2,2)$ & $(8,2,2)$ \\
\hline $782=2.17 .23$ & $28^{2}$ & {$[0,0,0]$} & {$[12,0,0]$} & {$[12,0,0]$} & {$[0,0,0]$} & $(12,2,2)$ & $(24,6,2)$ \\
\hline $1022=2.73 .7$ & $32^{2}$ & {$[16,0,0]$} & {$[0,0,0]$} & {$[0,0,0]$} & {$[0,0,0]$} & $(16,2,2)$ & $(32,8,2)$ \\
\hline $1246=2.89 .7$ & $21068856^{2}$ & {$[8,0,0]$} & {$[0,0,0]$} & {$[0,0,0]$} & {$[0,0,0]$} & $(8,2,2)$ & $(16,4,2)$ \\
\hline $1358=2.97 .7$ & $1732^{2}$ & {$[0,0,0]$} & {$[60,0,0]$} & {$[60,0,0]$} & {$[0,0,0]$} & $(12,2,2)$ & $(120,2,2)$ \\
\hline
\end{tabular}

\begin{tabular}{|c|c|c|c|c|c|c|c|}
\hline$d=2 p q$ & $x-1$ & $\mathcal{H}_{0} \mathcal{O}_{\mathbb{K}_{2}}$ & $\mathcal{H}_{1} \mathcal{O}_{\mathbb{K}_{2}}$ & $\mathcal{H}_{2} \mathcal{O}_{\mathbb{K}_{2}}$ & $\mathcal{H}_{1} \mathcal{H}_{2} \mathcal{O}_{\mathbb{K}_{2}}$ & $\mathrm{C} l(\mathbb{k})$ & $\mathbf{C} l\left(\mathbb{K}_{2}\right)$ \\
\hline $374=2.17 .11$ & $58^{2}$ & {$[0,2]$} & {$[0,0]$} & {$[0,0]$} & {$[0,0]$} & $(14,2,2)$ & $(28,4)$ \\
\hline $534=2.89 .3$ & $1918^{2}$ & {$[0,0]$} & {$[40,0]$} & {$[40,0]$} & {$[0,0]$} & $(10,2,2)$ & $(80,2)$ \\
\hline $1398=2.233 .3$ & $2206^{2}$ & {$[0,0]$} & {$[40,0]$} & {$[40,0]$} & {$[0,0]$} & $(10,2,2)$ & $(80,2)$ \\
\hline $2118=2.353 .3$ & $46^{2}$ & {$[60,12]$} & {$[0,0]$} & {$[0,0]$} & {$[0,0]$} & $(30,2,2)$ & $(120,24)$ \\
\hline $2694=2.449 .3$ & $2095718^{2}$ & {$[0,6,0]$} & {$[0,0,0]$} & {$[0,0,0]$} & {$[0,0,0]$} & $(30,2,2)$ & $(60,12,3)$ \\
\hline
\end{tabular}

(2) If $N\left(\epsilon_{2 p}\right)=1$ and $x+1, x-1$ are not squares in $\mathbb{N}$, then we are in the assumptions of the Proposition 4.3, since $N\left(\epsilon_{2 p}\right)=1$ yields that $p \equiv 1(\bmod 8)$. Moreover, Lemma 2.5 implies that $E_{\mathrm{k}}=\left\langle i, \epsilon_{2 p q}\right\rangle$.

(i) Assume $2 p(x+1)$ is a square in $\mathbb{N}$, hence, according to the Proposition 3.2. we have $E_{\mathbb{K}_{2}}=\left\langle i, \sqrt{i \epsilon_{q}}, \sqrt{i \epsilon_{2 p}}, \sqrt{i \epsilon_{2 p q}}\right\rangle$ and, according to the Theorem [5.2, there are two classes that capitulate in $\mathbb{K}_{2}$. So to prove the result, it suffices to show that $\mathcal{H}_{0}, \mathcal{H}_{1}$ and $\mathcal{H}_{0} \mathcal{H}_{1}$ do not capitulate in $\mathbb{K}_{2}$. If $\mathcal{H}_{0}$ (resp. $\left.\mathcal{H}_{1}, \mathcal{H}_{0} \mathcal{H}_{1}\right)$ capitulates in $\mathbb{K}_{2}$, then there exist $\alpha \in \mathbb{K}_{2}$ such that $\mathcal{H}_{0}=(\alpha)$ (resp. $\mathcal{H}_{1}=(\alpha)$, $\left.\mathcal{H}_{0} \mathcal{H}_{1}=(\alpha)\right)$, hence $\left(\alpha^{2}\right)=(1+i)$ (resp. $\left.\left(\alpha^{2}\right)=\left(\pi_{1}\right),\left(\alpha^{2}\right)=\left((1+i) \pi_{1}\right)\right)$. Consequently, $(1+i) \epsilon=\alpha^{2}$ (resp. $\left.\alpha^{2}=\pi_{1} \epsilon, \alpha^{2}=(1+i) \pi_{1} \epsilon\right)$ with some unit 
$\epsilon \in \mathbb{K}_{2}$; note that $\epsilon$ can be taken as follows $\epsilon=i^{a}{\sqrt{i \epsilon_{q}}}^{b}{\sqrt{i \epsilon_{2 p}}}^{c}{\sqrt{i \epsilon_{2 p q}}}^{d}$, where $a$, $b, c$ and $d$ are in $\{0,1\}$.

At first, let us show that the unit $\epsilon$ is neither real nor purely imaginary. In fact, if it is real (same proof if it is purely imaginary), then putting $\alpha=\alpha_{1}+i \alpha_{2}$, where $\alpha_{j} \in \mathbb{K}_{2}^{+}$, we get:

a. If $(1+i) \epsilon=\alpha^{2}$, then $\alpha_{1}^{2}-\alpha_{2}^{2}+2 i \alpha_{1} \alpha_{2}=\epsilon(1+i)$, hence $\left\{\begin{array}{l}\alpha_{1}^{2}-\alpha_{2}^{2}=\epsilon, \\ 2 \alpha_{1} \alpha_{2}=\epsilon,\end{array}\right.$

thus $\alpha_{1}^{2}-2 \alpha_{2} \alpha_{1}-\alpha_{2}^{2}=0$; therefore $\alpha_{1}=\alpha_{2}\left(1 \pm \sqrt{2}\right.$ ), and $\sqrt{2} \in \mathbb{K}_{2}^{+}$(for the case $\alpha^{2}=\pi_{1} \epsilon$, we get $\sqrt{p} \in \mathbb{K}_{2}^{+}$), which is absurd.

b. If $(1+i) \pi_{1} \epsilon=\alpha^{2}$, then $\alpha_{1}^{2}-\alpha_{2}^{2}+2 i \alpha_{1} \alpha_{2}=\epsilon(1+i) \pi_{1}$, hence $\left\{\begin{array}{l}\alpha_{1}^{2}-\alpha_{2}^{2}=\epsilon(e-4 f), \\ 2 \alpha_{1} \alpha_{2}=\epsilon(e+4 f),\end{array}\right.$ where $p=e^{2}+16 f^{2}$, since $p \equiv 1(\bmod 8)$. Thus

$$
4 \alpha_{1}^{4}-4 \epsilon(e-4 f) \alpha_{1}^{2}-\epsilon^{2}(e+4 f)^{2}=0
$$

from which we deduce that $\alpha_{1}^{2}=\frac{\epsilon}{2}[(e-4 f) \pm \sqrt{2 p}]$. As $\alpha_{1} \in \mathbb{K}_{2}^{+}$, so putting $\alpha_{1}=a+b \sqrt{2 p}$, where $a, b$ are in $\mathbb{Q}(\sqrt{q})$, we get the following unsolvable equation (in $\mathbb{Q}(\sqrt{q})$ ):

$$
16 a^{4}-8 \epsilon(e-4 f) a^{2}+2 p \epsilon^{2}=0,
$$

since its reduced discriminant is $\Delta^{\prime}=-16 \epsilon^{2}(e+4 f)^{2}<0$.

To this end, as $(1+i) \epsilon=\alpha^{2}$ (same proof for the other cases), then applying the norm $N_{\mathbb{K}_{2} / \mathbb{k}}$ we get that $(1+i)^{2} N_{\mathbb{K}_{2} / \mathbb{k}}(\epsilon)=N_{\mathbb{K}_{2} / \mathbb{k}}(\alpha)^{2}$, with $N_{\mathbb{K}_{2} / \mathbb{k}}(\epsilon) \in E_{\mathbb{k}}=$ $\left\langle i, \epsilon_{2 p q}\right\rangle$. Without loss of generality, one can take $N_{\mathbb{K}_{2} / \mathbb{k}}(\epsilon) \in\left\{ \pm 1, \pm i, \pm \epsilon_{2 p q}, \pm i \epsilon_{2 p q}\right\}$.

- As $N_{\mathbb{K}_{2} / \mathbb{k}}(\epsilon)$ is a square in $E_{\mathbb{k}}$, so $N_{\mathbb{K}_{2} / \mathbb{k}}(\epsilon) \notin\left\{ \pm i, \pm \epsilon_{2 p q}, \pm i \epsilon_{2 p q}\right\}$.

- If $N_{\mathbb{K}_{2} / \mathbb{k}}(\epsilon)= \pm 1$, then there exist $a, b, c$ and $d$ in $\{0,1\}$ such that $\epsilon=$ $i^{a}{\sqrt{i \epsilon_{q}}}^{b}{\sqrt{i \epsilon_{2 p}}}^{c}{\sqrt{i \epsilon_{2 p q}}}^{d}$ and $N_{\mathbb{K}_{2} / \mathbb{k}}(\epsilon)= \pm 1$, hence, $(-1)^{a} \epsilon_{2 p q}^{d} i^{b+c+d}= \pm 1$; so necessarily we must have $b=c$ and $d=0$. Therefore $\epsilon=i^{a+b}{\sqrt{\epsilon_{q} \epsilon_{2 p}}}^{b}$, which contradicts the fact that $\epsilon$ is not real or purely imaginary.

The following examples clarify this: the first table gives examples of the ideals $\mathcal{I}$, $\mathcal{H}_{0}$ and $\mathcal{H}_{1}$ which are not principal in $\mathbb{k}$, and gives the structures of the class groups of $\mathrm{k}$ and $\mathbb{K}_{2}$ respectively; whereas the second table gives the cases of capitulation of these ideals in $\mathbb{K}_{2}$.

\begin{tabular}{|l|l|l|l|l|l|l|l|}
\hline$d=2 p q$ & $2 p(x+1)$ & $\mathcal{I}$ & $\mathcal{I}^{2}$ & $\mathcal{H}_{0}$ & $\mathcal{H}_{1}$ & $\mathbf{C l}(\mathbb{k})$ & $\mathbf{C} l\left(\mathbb{K}_{2}\right)$ \\
\hline $582=2.97 .3$ & $194^{2}$ & {$[0,1,1]$} & {$[0,0,0]$} & {$[4,1,1]$} & {$[4,0,0]$} & $(8,2,2)$ & $(80,4,2)$ \\
\hline $646=2.17 .19$ & $102^{2}$ & {$[4,0,0]$} & {$[0,0,0]$} & {$[0,0,1]$} & {$[4,2,0]$} & $(8,4,2)$ & $(8,8,2,2)$ \\
\hline $2822=2.17 .83$ & $850^{2}$ & {$[12,1,0]$} & {$[0,0,0]$} & {$[0,0,1]$} & {$[12,0,0]$} & $(24,2,2)$ & $(48,12,2)$ \\
\hline $5654=2.257 .11$ & $178358^{2}$ & {$[28,1,0]$} & {$[0,0,0]$} & {$[0,0,1]$} & {$[28,0,0]$} & $(56,2,2)$ & $(224,8,4)$ \\
\hline $8854=2.233 .19$ & $9786^{2}$ & {$[0,0,1]$} & {$[0,0,0]$} & {$[60,0,1]$} & {$[60,0,0]$} & $(120,2,2)$ & $(120,8,2,2)$ \\
\hline $10806=2.1801 .3$ & $258569570^{2}$ & {$[0,1,1]$} & {$[0,0,0]$} & {$[24,1,0]$} & {$[24,0,0]$} & $(48,2,2)$ & $(48,48,6,2)$ \\
\hline
\end{tabular}

\begin{tabular}{|l|l|l|l|l|l|l|l|}
\hline$d=2 p q$ & $\mathcal{H}_{0} \mathcal{O}_{\mathbb{K}_{2}}$ & $\mathcal{H}_{1} \mathcal{O}_{\mathbb{K}_{2}}$ & $\mathcal{H}_{0} \mathcal{H}_{1} \mathcal{O}_{\mathbb{K}_{2}}$ & $\mathcal{I O}_{\mathbb{K}_{2}}$ & $\mathcal{H}_{1} \mathcal{I O}_{\mathbb{K}_{2}}$ & $\mathcal{H}_{0} \mathcal{I O}_{\mathbb{K}_{2}}$ & $\mathcal{H}_{0} \mathcal{H}_{1} \mathcal{I O}_{\mathbb{K}_{2}}$ \\
\hline $582=2.97 .3$ & {$[0,2,0]$} & {$[40,2,0]$} & {$[40,0,0]$} & {$[40,2,0]$} & {$[0,0,0]$} & {$[40,0,0]$} & {$[0,2,0]$} \\
\hline $646=2.17 .19$ & {$[0,4,1,1]$} & {$[4,4,1,1]$} & {$[4,0,0,0]$} & {$[0,0,0,0]$} & {$[0,0,1,1]$} & {$[4,0,1,1]$} & {$[0,4,0,0]$} \\
\hline $2822=2.17 .83$ & {$[0,6,0]$} & {$[24,6,0]$} & {$[24,0,0]$} & {$[0,6,0]$} & {$[24,0,0]$} & {$[0,0,0]$} & {$[24,6,0]$} \\
\hline $5654=2.257 .11$ & {$[0,4,0]$} & {$[112,0,0]$} & {$[112,4,0]$} & {$[112,0,0]$} & {$[0,0,0]$} & {$[112,4,0]$} & {$[0,4,0]$} \\
\hline $8854=2.233 .19$ & {$[60,4,1,1]$} & {$[0,4,0,0]$} & {$[60,0,1,1]$} & {$[60,0,1,1]$} & {$[60,4,1,1]$} & {$[0,4,0,0]$} & {$[0,0,0,0]$} \\
\hline
\end{tabular}




\begin{tabular}{|l|l|l|l|l|l|l|l|}
\hline$d=2 p q$ & $\mathcal{H}_{0} \mathcal{O}_{\mathbb{K}_{2}}$ & $\mathcal{H}_{1} \mathcal{O}_{\mathbb{K}_{2}}$ & $\mathcal{H}_{0} \mathcal{H}_{1} \mathcal{O}_{\mathbb{K}_{2}}$ & $\mathcal{I O}_{\mathbb{K}_{2}}$ & $\mathcal{H}_{1} \mathcal{I} \mathcal{O}_{\mathbb{K}_{2}}$ & $\mathcal{H}_{0} \mathcal{I O}_{\mathbb{K}_{2}}$ & $\mathcal{H}_{0} \mathcal{H}_{1} \mathcal{I} \mathcal{O}_{\mathbb{K}_{2}}$ \\
\hline $10806=2.1801 .3$ & {$[24,24,0,1]$} & {$[24,24,0,0]$} & $0,0,0,1]$ & {$[0,0,0,0]$} & {$[24,24,0,0]$} & {$[24,24,0,1]$} & $0,0,0,1]$ \\
\hline
\end{tabular}

(ii) Assume $p(x \pm 1)$ is a square in $\mathbb{N}$, hence, according to the Proposition 3.2. we have $E_{\mathbb{K}_{2}}=\left\langle i, \sqrt{i \epsilon_{q}}, \sqrt{i \epsilon_{2 p}}, \sqrt{\epsilon_{2 p q}}\right\rangle$. Thus proceeding as in the case (i) we prove that $\mathcal{H}_{1}, \mathcal{H}_{0}$ and $\mathcal{H}_{0} \mathcal{H}_{1}$ do not capitulate in $\mathbb{K}_{2}$. The following examples illustrate these results.

(a) First case: $p(x+1)$ is a square in $\mathbb{N}$. The first table gives examples of the ideals $\mathcal{I}, \mathcal{H}_{0}$ and $\mathcal{H}_{1}$ which are not principal in $\mathbb{k}$, and gives the structures of the class groups of $\mathbb{k}$ and $\mathbb{K}_{2}$ respectively; whereas the second table gives the cases of capitulation of these ideals in $\mathbb{K}_{2}$.

\begin{tabular}{|l|l|l|l|l|l|l|l|}
\hline$d=2 p q$ & $p(x+1)$ & $\mathcal{I}$ & $\mathcal{I}^{2}$ & $\mathcal{H}_{0}$ & $\mathcal{H}_{1}$ & $\mathrm{C} l(\mathrm{k})$ & $\mathrm{C} l\left(\mathbb{K}_{2}\right)$ \\
\hline $3358=2.73 .23$ & $217248^{2}$ & {$[4,0,0]$} & {$[0,0,0]$} & {$[0,2,1]$} & {$[0,2,0]$} & $(8,4,2)$ & $(96,8,2,2)$ \\
\hline $3502=2.17 .103$ & $447916^{2}$ & {$[2,2,0]$} & {$[0,0,0]$} & {$[2,0,1]$} & {$[0,2,0]$} & $(4,4,2)$ & $(20,4,2,2,2)$ \\
\hline $6014=2.97 .31$ & $388^{2}$ & {$[12,0,0]$} & {$[0,0,0]$} & {$[0,4,1]$} & {$[12,4,0]$} & $(24,8,2)$ & $(240,24,2,2)$ \\
\hline $9118=2.97 .47$ & $11181384^{2}$ & {$[4,0,0]$} & {$[0,0,0]$} & {$[4,0,1]$} & {$[0,2,0]$} & $(8,4,2)$ & $(20,20,4,2,2)$ \\
\hline
\end{tabular}

\begin{tabular}{|l|l|l|l|l|l|l|l|}
\hline$d=2 p q$ & $\mathcal{H}_{0} \mathcal{O}_{\mathbb{K}_{2}}$ & $\mathcal{H}_{1} \mathcal{O}_{\mathbb{K}_{2}}$ & $\mathcal{H}_{0} \mathcal{H}_{1} \mathcal{O}_{\mathbb{K}_{2}}$ & $\mathcal{I O}_{\mathbb{K}_{2}}$ & $\mathcal{H}_{1} \mathcal{I O}_{\mathbb{K}_{2}}$ & $\mathcal{H}_{0} \mathcal{I O}_{\mathbb{K}_{2}}$ & $\mathcal{H}_{0} \mathcal{H}_{1} \mathcal{I} \mathcal{O}_{\mathbb{K}}$ \\
\hline $\begin{array}{l}3358= \\
2.73 .23\end{array}$ & {$[48,4,0,0]$} & {$[48,0,0,0]$} & {$[0,4,0,0]$} & {$[0,4,0,0]$} & {$[48,4,0,0]$} & {$[48,0,0,0]$} & {$[0,0,0,0]$} \\
\hline $\begin{array}{l}3502= \\
2.17 .103\end{array}$ & {$[0,0,1,0,0]$} & {$[0,2,0,0,0]$} & {$[0,2,1,0,0$} & {$[0,2,0,0,0]$} & {$[0,0,0,0,0]$} & {$[0,2,1,0,0]$} & {$[0,0,1,0,0$} \\
\hline $\begin{array}{l}6014= \\
2.97 .31\end{array}$ & & & & & & & \\
\hline $\begin{array}{l}9118= \\
2.97 .47\end{array}$ & {$[120,12,0,0]$} & {$[120,0,0,0]$} & {$[0,12,0,0]$} & {$[120,12,0,0$} & {$[0,12,0,0]$} & {$[0,0,0,0]$} & {$[120,0,0,0$} \\
\hline
\end{tabular}

(b) Second case: $p(x-1)$ is a square in $\mathbb{N}$. The first table gives examples of the ideals $\mathcal{I}, \mathcal{H}_{0}$ and $\mathcal{H}_{1}$ which are not principal in $\mathbb{k}$, and gives the structures of the class groups of $\mathbb{k}$ and $\mathbb{K}_{2}$ respectively; whereas the second table gives the cases of capitulation of these ideals in $\mathbb{K}_{2}$.

\begin{tabular}{|l|l|l|l|l|l|l|l|}
\hline$d=2 p q$ & $p(x-1)$ & $\mathcal{I}$ & $\mathcal{I}^{2}$ & $\mathcal{H}_{0}$ & $\mathcal{H}_{1}$ & $\mathbf{C} l(\mathbb{k})$ & $\mathbf{C} l\left(\mathbb{K}_{2}\right)$ \\
\hline $438=2.73 .3$ & 21316 & {$[0,1,1]$} & {$[0,0,0]$} & {$[2,1,1]$} & {$[2,0,0]$} & $(4,2,2)$ & $(32,2,2,2)$ \\
\hline $2022=2.337 .3$ & 454276 & {$[6,1,0]$} & {$[0,0,0]$} & {$[0,0,1]$} & {$[6,0,0]$} & $(12,2,2)$ & $(48,24,2)$ \\
\hline $2598=2.433 .3$ & 749956 & {$[6,1,1]$} & {$[0,0,0]$} & {$[0,1,1]$} & {$[6,0,0]$} & $(12,2,2)$ & $(132,4,4)$ \\
\hline $5622=2.937 .3$ & 3511876 & {$[0,2,1]$} & {$[0,0,0]$} & {$[0,0,1]$} & {$[8,2,0]$} & $(16,4,2)$ & $(224,8,4)$ \\
\hline
\end{tabular}

\begin{tabular}{|l|l|l|l|l|l|l|l|}
\hline$d=2 p q$ & $\mathcal{H}_{0} \mathcal{O}_{\mathbb{K}_{2}}$ & $\mathcal{H}_{1} \mathcal{O}_{\mathbb{K}_{2}}$ & $\mathcal{H}_{0} \mathcal{H}_{1} \mathcal{O}_{\mathbb{K}_{2}}$ & $\mathcal{I O}_{\mathbb{K}_{2}}$ & $\mathcal{H}_{1} \mathcal{I O}_{\mathbb{K}_{2}}$ & $\mathcal{H}_{0} \mathcal{I O}_{\mathbb{K}_{2}}$ & $\mathcal{H}_{0} \mathcal{H}_{1} \mathcal{I O}_{\mathbb{K}_{2}}$ \\
\hline $438=2.73 .3$ & {$[16,1,1,1]$} & {$[0,1,1,1]$} & {$[16,0,0,0]$} & {$[0,1,1,1]$} & {$[0,0,0,0]$} & {$[16,0,0,0$} & {$[16,1,1,1]$} \\
\hline $2022=2.337 .3$ & {$[24,12,0]$} & {$[0,12,0]$} & {$[24,0,0]$} & {$[24,12,0]$} & {$[24,0,0]$} & {$[0,0,0]$} & {$[0,12,0]$} \\
\hline $2598=2.433 .3$ & {$[66,2,0]$} & {$[0,2,2]$} & {$[66,0,2]$} & {$[66,0,2]$} & {$[66,2,0]$} & {$[0,2,2]$} & {$[0,0,0]$} \\
\hline $5622=2.937 .3$ & {$[112,4,0]$} & {$[112,0,0]$} & {$[0,4,0]$} & {$[0,0,0]$} & {$[112,0,0]$} & {$[112,4,0]$} & {$[0,4,0]$} \\
\hline
\end{tabular}

(3) Suppose that $N\left(\epsilon_{2 p}\right)=-1$. Prove that $\mathcal{H}_{0} \mathcal{H}_{1}$ and $\mathcal{H}_{0} \mathcal{H}_{2}$ capitulate in $\mathbb{K}_{2}$. Put $\epsilon_{2 p}=a+b \sqrt{2 p}$, then $a^{2}+1=2 b^{2} p$, hence by the decomposition uniqueness in $\mathbb{Z}[i]$ there exist $b_{1}$ and $b_{2}$ in $\mathbb{Z}[i]$ such that:

$$
\left\{\begin{array} { l } 
{ a \pm i = b _ { 1 } ^ { 2 } ( 1 + i ) \pi _ { 1 } , } \\
{ a \mp i = b _ { 2 } ^ { 2 } ( 1 - i ) \pi _ { 2 } , }
\end{array} \quad \text { or } \left\{\begin{array}{l}
a \pm i=i(1+i) b_{1}^{2} \pi_{1}, \\
a \mp i=-i(1-i) b_{2}^{2} \pi_{2},
\end{array} \quad \text { with } b=b_{1} b_{2} .\right.\right.
$$

Consequently $\sqrt{\epsilon_{2 p}}=\frac{1}{2}\left(b_{1}(1+i) \sqrt{(1 \pm i) \pi_{1}}+b_{2}(1-i) \sqrt{(1 \mp i) \pi_{2}}\right)$, hence $(1 \pm$ i) $\pi_{1} \epsilon_{2 p}$ and $(1 \mp i) \pi_{2} \epsilon_{2 p}$ are squares in $\mathbb{K}_{2}$. Thus $\left(\alpha^{2}\right)=\left((1 \pm i) \pi_{1}\right)$ and $\left(\beta^{2}\right)=$ $\left((1 \mp i) \pi_{2}\right)$, with some $\alpha, \beta$ in $\mathbb{K}_{2}$. Therefore $\mathcal{H}_{0} \mathcal{H}_{1}=(\alpha)$ and $\mathcal{H}_{0} \mathcal{H}_{2}=(\beta)$ i.e. 
$\mathcal{H}_{0} \mathcal{H}_{1}$ and $\mathcal{H}_{0} \mathcal{H}_{2}$ capitulate in $\mathbb{K}_{2}$.

(i) If $x \pm 1$ is a square in $\mathbb{N}$, then Lemma 4.1 yields that $\mathcal{H}_{1} \mathcal{H}_{2}, \mathcal{H}_{0} \mathcal{H}_{1}$ and $\mathcal{H}_{0} \mathcal{H}_{2}$ are not principal in $\mathrm{k}$, hence the result.

(ii) If $x+$ and $x-1$ are not squares in $\mathbb{N}$, then Lemma 4.1 yields that $\left[\mathcal{H}_{0} \mathcal{H}_{1}\right]=$ $\left[\mathcal{H}_{0} \mathcal{H}_{2}\right]$, hence the result.

5.3. Capitulation in $\mathbb{K}_{3}$. Let $\mathbb{K}_{3}=\mathbb{k}_{k}(\sqrt{2})=\mathbb{Q}(\sqrt{2}, \sqrt{p q}, i)$ and put $\epsilon_{p q}=a+$ $b \sqrt{p q}, \epsilon_{2 p q}=x+y \sqrt{2 p q}$. Let $Q_{\mathbb{K}_{3}}$ denote the unit index of $\mathbb{K}_{3}$.

Theorem 5.7. Keep the notations and hypotheses previously mentioned.

(1) If both of $x \pm 1$ and $a \pm 1$ are squares in $\mathbb{N}$, then

(a) If $Q_{\mathbb{K}_{3}}=2$, then $\kappa_{\mathbb{K}_{3}}=\left\langle\left[\mathcal{H}_{0}\right]\right\rangle$.

(b) If $Q_{\mathbb{K}_{3}}=1$, then $\kappa_{\mathbb{K}_{3}}=\left\langle\left[\mathcal{H}_{0}\right],\left[\mathcal{H}_{1} \mathcal{H}_{2}\right]\right\rangle$.

(2) If $x \pm 1$ is a square in $\mathbb{N}$ and $a+1, a-1$ are not, then $\kappa_{\mathbb{K}_{3}}=\left\langle\left[\mathcal{H}_{0}\right],\left[\mathcal{H}_{1} \mathcal{H}_{2}\right]\right\rangle$.

(3) If $a \pm 1$ is a square in $\mathbb{N}$ and $x+1, x-1$ are not, then there exist an unambiguous ideal $\mathcal{I}$ in $\mathrm{k} / \mathbb{Q}(i)$ of order 2 such that $\kappa_{\mathbb{K}_{3}}=\left\langle\left[\mathcal{H}_{0}\right],[\mathcal{I}]\right\rangle$ or $\left\langle\left[\mathcal{H}_{0}\right],\left[\mathcal{H}_{1} \mathcal{I}\right]\right\rangle$.

(4) If $x+1, x-1, a+1$ and $a-1$ are note squares in $\mathbb{N}$, then $\kappa_{\mathbb{K}_{3}}=\left\langle\left[\mathcal{H}_{0}\right]\right\rangle$.

Proof. As $N\left(\epsilon_{2}\right)=-1$, then $\sqrt{(1+i) \epsilon_{2}}=\frac{1}{2}(2+(1+i) \sqrt{2})$. Hence there exist $\beta \in \mathbb{K}_{3}$ such that $\mathcal{H}_{0}^{2}=(1+i)=\left(\beta^{2}\right)$, therefore $\mathcal{H}_{0}$ capitulates in $\mathbb{K}_{3}$.

(1) Assume $x \pm 1$ and $a \pm 1$ are squares in $\mathbb{N}$.

(a) If $Q_{\mathbb{K}_{3}}=2$, then by the Theorem [5.2, $\left|\kappa_{\mathbb{K}_{3}}\right|=2$, hence $\kappa_{\mathbb{K}_{3}}=\left\langle\left[\mathcal{H}_{0}\right]\right\rangle$.

(b) If $Q_{\mathbb{K}_{3}}=1$, then by the Theorem $5.2,\left|\kappa_{\mathbb{K}_{3}}\right|=4$. Since $a \pm 1$ is a square in $\mathbb{N}$, so the Lemma 5.5 yields that $p \equiv 1(\bmod 8)$. Therefore the Proposition 4.3 implies that

$$
\operatorname{Am}(\mathbb{k} / \mathbb{Q}(i))=\operatorname{Am}_{s}(\mathbb{k} / \mathbb{Q}(i))=\left\langle\left[\mathcal{H}_{0}\right],\left[\mathcal{H}_{1}\right],\left[\mathcal{H}_{2}\right]\right\rangle .
$$

Proceeding as in the proof of Theorem [5.6)(2), we show that $\mathcal{H}_{1}$ and $\mathcal{H}_{2}$ do not capitulate in $\mathbb{K}_{3}$. On the other hand, as $\left|\kappa_{\mathbb{K}_{3}}\right|=4$ and $\kappa_{\mathbb{K}_{3}} \subseteq \operatorname{Am}(\mathbb{k} / \mathbb{Q}(i))$, so necessarily $\mathcal{H}_{1} \mathcal{H}_{2}$ capitulate in $\mathbb{K}_{3}$. Finally, Lemma 4.1 yields that $\mathcal{H}_{1} \mathcal{H}_{2}, \mathcal{H}_{0}$ and $\mathcal{H}_{0} \mathcal{H}_{1} \mathcal{H}_{2}$ are not principal in $\mathrm{k}$. Thus the result.

(2) Assume $x \pm 1$ is a square in $\mathbb{N}$ and $a+1, a-1$ are not. As $\mathcal{H}_{0}$ capitulates in $\mathbb{K}_{3}$ and $\left|\kappa_{\mathbb{K}_{3}}\right|=4$ (Theorem [5.2), it suffices to prove that $\mathcal{H}_{1} \mathcal{H}_{2}$ capitulates in $\mathbb{K}_{3}$. According to the proof of Proposition 3.3 , we have $p \epsilon_{p q}$ is a square in $\mathbb{K}_{3}$; hence there exist $\alpha$ in $\mathbb{K}_{3}$ such that $(p)=\left(\alpha^{2}\right)$, so $\mathcal{H}_{1} \mathcal{H}_{2}=(\alpha)$. Thus the result.

(3) If $a \pm 1$ is a square in $\mathbb{N}$ and $x+1, x-1$ are not, then Lemma 5.5 implies that $p \equiv 1(\bmod 8)$; hence we are in the hypotheses of the Proposition 4.3. On the other hand, from the Lemma 4.1 we get $\left[\mathcal{H}_{1}\right]=\left[\mathcal{H}_{2}\right]$. Therefore, proceeding as in the proof of Theorem 5.6, we show that $\mathcal{H}_{1}$ does not capitulate in $\mathbb{K}_{3}$. The following examples clarify the two cases of capitulation:

\begin{tabular}{|c|c|c|c|c|c|c|}
\hline$d=2 p q$ & $\mathcal{H}_{0} \mathcal{O}_{\mathbb{K}_{3}}$ & $\mathcal{H}_{1} \mathcal{O}_{\mathbb{K}_{3}}$ & $\mathcal{I O}_{\mathbb{K}_{3}}$ & $\mathcal{H}_{1} \mathcal{I} \mathcal{O}_{\mathbb{K}_{3}}$ & $\mathbf{C l}(\mathbb{k})$ & $\mathbf{C l}\left(\mathbb{K}_{2}\right)$ \\
\hline $582=2.97 .3$ & {$[0,0,0]$} & {$[8,2,0]$} & {$[0,0,0]$} & {$[8,2,0]$} & $(8,2,2)$ & $(16,4,2)$ \\
\hline $2006=2.17 .59$ & {$[0,0,0]$} & {$[24,0,0]$} & {$[24,0,0]$} & {$[0,0,0]$} & $(24,2,2)$ & $(48,4,2)$ \\
\hline $2454=2.409 .3$ & {$[0,0,0]$} & {$[16,0,0]$} & {$[16,0,0]$} & {$[0,0,0]$} & $(16,2,2)$ & $(32,4,2)$ \\
\hline $2742=2.457 .3$ & {$[0,0,0]$} & {$[48,2,0]$} & {$[0,0,0]$} & {$[48,2,0]$} & $(16,2,2)$ & $(96,4,2)$ \\
\hline
\end{tabular}


(4) Suppose that $x+1, x-1, a+1$ and $a-1$ are not squares in $\mathbb{N}$, then $\left|\kappa_{\mathbb{K}_{3}}\right|=2$ (Theorem 5.2 ). Thus $\kappa_{\mathbb{K}_{3}}=\left\langle\left[\mathcal{H}_{0}\right]\right\rangle$.

From theorems 5.3, 5.6 and 5.7 we deduce the following theorem.

Theorem 5.8. Let $\mathbb{k}=\mathbb{Q}(\sqrt{2 p q}, i)$, where $p \equiv-q \equiv 1(\bmod 4)$ are different primes, and $\mathbb{k}^{(*)}$ its genus field. Put $\epsilon_{2 p q}=x+y \sqrt{2 p q}$ and $\epsilon_{p q}=a+b \sqrt{p q}$.

(1) If $x \pm 1$ is a square in $\mathbb{N}$, then $\left\langle\left[\mathcal{H}_{0}\right],\left[\mathcal{H}_{1}\right],\left[\mathcal{H}_{2}\right]\right\rangle \subseteq \kappa_{\mathbb{k}^{(*)}}$.

(2) If $x+1$ and $x-1$ are not squares in $\mathbb{N}$, then

(a) If $N\left(\epsilon_{2 p}\right)=1$ or $a \pm 1$ is a square in $\mathbb{N}$, then there exists an unambiguous ideal $\mathcal{I}$ in $\mathrm{k} / \mathbb{Q}(i)$ of order 2 such that: $\left\langle\left[\mathcal{H}_{0}\right],\left[\mathcal{H}_{1}\right],[\mathcal{I}]\right\rangle \subseteq \kappa_{\mathrm{lk}^{(*)}}$.

(b) Else $\left\langle\left[\mathcal{H}_{0}\right],\left[\mathcal{H}_{1}\right]\right\rangle \subseteq \kappa_{\mathbb{k}}(*)$.

Theorem 5.8 implies the following corollary:

Corollary 5.9. Let $\mathrm{k}=\mathbb{Q}(\sqrt{2 p q}, i)$, where $p \equiv-q \equiv 1(\bmod 4)$ are different primes. Let $\mathbb{k}^{(*)}$ be the genus field of $\mathbb{k}$ and $\mathrm{A} m_{s}(\mathrm{k} / \mathbb{Q}(i))$ be the group of the strongly ambiguous class of $\mathbb{k} / \mathbb{Q}(i)$, then $\mathrm{A} m_{s}(\mathbb{k} / \mathbb{Q}(i)) \subseteq \kappa_{\mathbb{k}(*)}$.

\section{Application}

Let $p \equiv-q \equiv 1(\bmod 4)$ be different primes such that $p \equiv 1(\bmod 8), q \equiv 3$ $(\bmod 8)$ and $\left(\frac{p}{q}\right)=-1$. Hence, according to [3], $\mathbf{C} l_{2}(\mathbb{k})$ is of type $(2,2,2)$. Therefore, under these assumptions, $\mathbf{C l} l_{2}(\mathbb{k})=\mathrm{A} m_{s}(\mathrm{k} / \mathbb{Q}(i))=\left\langle\left[\mathcal{H}_{0}\right],\left[\mathcal{H}_{1}\right],\left[\mathcal{H}_{2}\right]\right\rangle$ (see [5]). To continue we need the following result.

Lemma 6.1. Let $\epsilon_{2 p q}=x+y \sqrt{2 p q}$ (resp. $\left.\epsilon_{p q}=a+b \sqrt{p q}\right)$ denote the fundamental unit of $\mathbb{Q}(\sqrt{2 p q})$ (resp. $\mathbb{Q}(\sqrt{p q}))$. Then

(1) $x-1$ is a square in $\mathbb{N}$.

(2) $a-1$ is a square in $\mathbb{N}$.

Proof. (1) By Lemma 2.2 and according to the decomposition uniqueness in $\mathbb{Z}$, there are six cases to discus: $x \pm 1$ or $p(x \pm 1)$ or $2 p(x \pm 1)$ is a square in $\mathbb{N}$.

a. If $x+1$ is a square in $\mathbb{N}$, then $\left\{\begin{array}{l}x+1=y_{1}^{2}, \\ x-1=2 p q y_{2}^{2},\end{array}\right.$

hence $1=\left(\frac{x+1}{q}\right)=\left(\frac{x-1+2}{q}\right)=\left(\frac{2}{q}\right)$, which contradicts the fact that $\left(\frac{2}{q}\right)=$ -1 .

b. If $p(x \pm 1)$ is a square in $\mathbb{N}$, then $\left\{\begin{array}{l}x \pm 1=p y_{1}^{2}, \\ x \mp 1=2 q y_{2}^{2},\end{array}\right.$

hence $\left(\frac{2 q}{p}\right)=\left(\frac{x \mp 1}{p}\right)=\left(\frac{x \pm 1 \mp 2}{p}\right)=\left(\frac{2}{p}\right)$, thus $\left(\frac{q}{p}\right)=1$. Which is false, since $\left(\frac{p}{q}\right)=-1$. 
c. If $2 p(x+1)$ is a square in $\mathbb{N}$, then $\left\{\begin{array}{l}x+1=p y_{1}^{2}, \\ x-1=2 q y_{2}^{2},\end{array}\right.$

hence $\left(\frac{2 p}{q}\right)=\left(\frac{x+1}{q}\right)=\left(\frac{x-1+2}{q}\right)=\left(\frac{2}{q}\right)$, which leads to the contradiction $\left(\frac{q}{p}\right)=1$.

d. If $2 p(x-1)$ is a square in $\mathbb{N}$, then $\left\{\begin{array}{l}x-1=p y_{1}^{2}, \\ x+1=2 q y_{2}^{2}\end{array}\right.$,

hence $\left(\frac{q}{p}\right)=\left(\frac{x+1}{p}\right)=\left(\frac{x-1+2}{p}\right)=\left(\frac{2}{p}\right)=1$, which is false.

Consequently, the only case which is possible is: $x-1$ is a square in $\mathbb{N}$.

(2) Proceeding similarly, we show that $a-1$ is a square in $\mathbb{N}$.

Theorem 6.2. Let $\mathbb{k}=\mathbb{Q}(\sqrt{2 p q}, i)$, where $p \equiv-q \equiv 1(\bmod 4)$ are different primes satisfying the conditions $p \equiv 1(\bmod 8), q \equiv 3(\bmod 8)$ and $\left(\frac{p}{q}\right)=-1$. Put $\mathbb{K}_{1}=\mathbb{k}(\sqrt{p}), \mathbb{K}_{2}=\mathbb{k}_{(}(\sqrt{q})$ and $\mathbb{K}_{3}=\mathbb{k}_{(}(\sqrt{2})$. Let $\mathbb{k}^{(*)}$ denote the absolute genus field of $\mathbb{k}$ and $(\mathbb{k} / \mathbb{Q}(i))^{*}$ its relative genus field over $\mathbb{Q}(i)$.

(1) $\mathbb{k}^{(*)} \varsubsetneqq(\mathbb{k} / \mathbb{Q}(i))^{*}$.

(2) $\kappa_{\mathbb{K}_{1}}=\left\langle\left[\mathcal{H}_{1}\right],\left[\mathcal{H}_{2}\right]\right\rangle$.

(3) Denote by $\epsilon_{2 p}$ the fundamental unit of $\mathbb{Q}(\sqrt{2 p})$, so:

(a) If $N\left(\epsilon_{2 p}\right)=1$, then $\kappa_{\mathbb{K}_{2}}=\left\langle\left[\mathcal{H}_{1}\right],\left[\mathcal{H}_{2}\right]\right\rangle$ or $\left\langle\left[\mathcal{H}_{0}\right]\right.$, $\left.\left[\mathcal{H}_{1} \mathcal{H}_{2}\right]\right\rangle$.

(b) Else, $\kappa_{\mathbb{K}_{2}}=\left\langle\left[\mathcal{H}_{0} \mathcal{H}_{1}\right],\left[\mathcal{H}_{0} \mathcal{H}_{2}\right]\right\rangle$

(4) Denote by $Q_{\mathbb{K}_{3}}$ the unit index of $\mathbb{K}_{3}$, so:

(a) If $Q_{\mathbb{K}_{3}}=1$, then $\kappa_{\mathbb{K}_{3}}=\left\langle\left[\mathcal{H}_{0}\right],\left[\mathcal{H}_{1} \mathcal{H}_{2}\right]\right\rangle$.

(b) If $Q_{\mathbb{K}_{3}}=2$, then $\kappa_{\mathbb{K}_{3}}=\left\langle\left[\mathcal{H}_{0}\right]\right\rangle$

(5) $\kappa_{\mathbb{k}(*)}=A m_{s}(\mathbb{k} / \mathbb{Q}(i))=\mathrm{Cl}_{2}(\mathbb{k})$.

Proof. (1) From Lemma 6.1 we have $x-1$ is a square in $\mathbb{N}$. Then Proposition 4.3 yields the first assertion.

(2) From Lemma 6.1, we have $x-1$ is a square in $\mathbb{N}$. Then Theorem 5.3(1) yields the second assertion.

(3) From Lemma 6.1, we have $x-1$ is a square in $\mathbb{N}$. Therefore

(a) If $N\left(\epsilon_{2 p}\right)=1$, then Theorem 5.6(1) yields the result.

(b) If $N\left(\epsilon_{2 p}\right)=-1$, then Theorem 5.6 (3) yields the result.

(4) As $x-1$ and $a-1$ are squares in $\mathbb{N}$ (Lemma 6.1), so Theorem 5.7(1) yields the result.

(5) As $p \equiv 1(\bmod 8)$, so from Proposition 4.3 we get $\mathrm{A} m_{s}(\mathrm{k} / \mathbb{Q}(i))=\left\langle\left[\mathcal{H}_{0}\right],\left[\mathcal{H}_{1}\right],\left[\mathcal{H}_{2}\right]\right\rangle$. Hence $\mathrm{A} m_{s}(\mathbb{k} / \mathbb{Q}(i))=\mathrm{Cl}_{2}(\mathbb{k})$. The assertions (2), (3) and (4) imply that $\kappa_{\mathbb{k}}(*)=\mathrm{A} m_{s}(\mathbb{k} / \mathbb{Q}(i))=\mathrm{Cl}_{2}(\mathbb{k})$.

\section{ACKNOWLEDGEMENT}

We would like to thank the unknown referee for his several helpful suggestions and for calling our attention to the missing details. 


\section{REFERENCES}

[1] A. Azizi, Sur la capitulation des 2-classes d'idéaux de $\mathbb{k}=\mathbb{Q}(\sqrt{2 p q}, i)$, où $p \equiv-q \equiv 1$ (mod 4), Acta. Arith. 94 (2000), 383-399, Zbl 0953.11033, MR 1779950.

[2] A. Azizi, Unités de certains corps de nombres imaginaires et abéliens sur $\mathbb{Q}$, Ann. Sci. Math. Québec 23 (1999), no 1, 15-21, Zbl 1041.11072, MR 1721726.

[3] A. Azizi and M. Taous, Détermination des corps $\mathbf{k}=\mathbb{Q}(\sqrt{d}, \sqrt{-1})$ dont les 2-groupes de classes sont de type $(2,4)$ ou $(2,2,2)$, Rend. Istit. Mat. Univ. Trieste. 40 (2008), 93-116, Zbl 1215.11107, MR2583453.

[4] A. Azizi, A. Zekhnini and M. Taous, On the unramified quadratic and biquadratic extensions of the field $\mathbb{Q}(\sqrt{d}, i)$, IJA, Volume 6, No. 24 (2012), 1169-1173, Zbl 1284.11142, MR2974674.

[5] A. Azizi, A. Zekhnini and M. Taous, On the generators of the 2-class group of the field $\mathbf{k}=\mathbb{Q}(\sqrt{d}, i)$, IJPAM, Volume 81, No. 5 (2012), 773-784.

[6] A. Azizi, A. Zekhnini and M. Taous, On the strongly ambiguous classes of $\mathbb{k} / \mathbb{Q}(i)$ where $\mathbf{k}=\mathbb{Q}\left(\sqrt{2 p_{1} p_{2}}, i\right)$, Asian-Eur. J. Math. 7 (2014), no. 1, Zbl 1292.11119, MR3189588.

[7] A. Azizi, A. Zekhnini and M. Taous, Structure of $\mathrm{Gal}\left(\mathrm{k}_{2}^{(2)} / \mathbb{k}\right)$ for some fields $\mathbb{k}=$ $\mathbb{Q}\left(\sqrt{2 p_{1} p_{2}}, i\right)$ with $\mathbf{C l}_{2}(\mathrm{k}) \simeq(2,2,2)$, Abh. Math. Sem. Univ. Hamburg, Vol 84, 2 (2014), 203-231, MR3267742.

[8] C. Chevalley, Sur la théorie du corps de classes dans les corps finis et les corps locaux, J. Fac. Sc. Tokyo, Sect. 1, t.2, (1933), 365-476, Zbl 0008.05301.

[9] F. Lemmermeyer, The ambiguous class number formula revisited, J. of the Ramanujan Math. Soc. Volume 28, 4, 415-421, MR3158989.

[10] F. P. Heider and B. Schmithals, Zur kapitulation der idealklassen in unverzweigten primzyklischen erweiterungen, J. Reine Angew. Math. 366 (1982), 1-25, Zbl 0505.12016, MR 0671319 .

[11] F. Terada, A principal ideal theorem in the genus fields, Tohoku Math. J. 23, No. 2 (1971), 697-718, Zbl0243.12003, MR0306158.

[12] G. Gras, Class field theory, from theory to practice, Springer Verlag (2003), Zbl 1019.11032, MR 1941965.

[13] H. Furuya, Principal ideal theorems in the genus field for absolutely abelian extensions, J. Number Theory 9, (1977), 4-15, Zbl 0347.12006, MR0429820.

[14] H. Hasse, Über die Klassenzahl abelscher Zahlkörper, Berlin, Akademie-Verlag, (1952), Zbl46.260, MR 0049239.

[15] H. Wada, On the class number and the unit group of certain algebraic number fields, J. Fac. Univ. Tokyo Sect. I 13 (1966), 201-209, Zbl 0158.30103, MR 0214565.

[16] M. Hirabayashi and K. Yoshino, Unit indices of imaginary abelian number fields of type $(2,2,2)$, J. N. Theory, 34, No. 3 (1990), 346-361, Zbl 0705.11065, MR1049510.

[17] P. J. Sime, On the ideal class group of real biquadratic fields, Trans. Am. Math. Soc. 347 No. 12 (1995), 4855-4876, Zbl 0847.11060, MR 1333398.

[18] S. Kuroda, Über den Dirichletschen Körper, J. Fac. Sci. Imp. Univ. Tokyo, sec I, vol IV, part 5, (1943), 383-406, Zbl 0061.05901, MR 0021031.

[19] S. Louboutin. Hasse unit indices of dihedral octic CM-fields, Math. Nachr. 215 (2000), 107-113, Zbl 0972.11105, MR 1768197.

[20] T. Kubota, Über den bizyklischen biquadratischen Zahlkörper, Nagoya Math. J. 10 (1956), 65-85, Zbl 0074.03001, MR 0083009.

[21] T. M. McCall, C. J. Parry and R. R. Ranalli, Imaginary bicyclic biquadratic fields with cyclic 2-class group, J. Number Theory 53, 88-99 (1995), Zbl 0831.11059, MR 1344833.

Authors' addresses:

Abdelmalek Azizi and Abdelkader Zekhnini, Mohammed First University, Mathematics Department, Sciences Faculty, Oujda, Morocco. 
e-mail: abdelmalekazizi@yahoo.fr

e-mail: zekha1@yahoo.fr.

Mohammed Taous, Moulay Ismail University, Mathematics Department, Sciences and Techniques Faculty, Errachidia, Morocco.

e-mail: taousm@hotmail.com

Abdelmalek Azizi and Abdelkader Zekhnini: DÃ@partement de Mathã@ematiques, Facultã@ Des Sciences, Universitã C Mohammed 1, Oujda, Morocco

E-mail address: abdelmalekazizi@yahoo.fr

E-mail address: zekha1@yahoo.fr

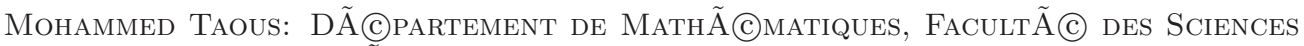
et Techniques, Universitã (C) Moulay Ismail, Errachidia, Morocco

E-mail address: taousm@hotmail.com 